\title{
Analysis of RIM Expression and Function at Mouse Photoreceptor Ribbon Synapses
}

\author{
Martina Löhner, ${ }^{1}$ @Norbert Babai, ${ }^{1}$ Tanja Müller, ${ }^{1}$ @Kaspar Gierke, ${ }^{1}$ Jenny Atorf, ${ }^{2}$ Anneka Joachimsthaler, ${ }^{2}$ \\ Angela Peukert, ${ }^{1}{ }^{\circ}$ Henrik Martens, ${ }^{3}$ Andreas Feigenspan, ${ }^{1}$ ㅇa Kremers, ${ }^{2}$ @Susanne Schoch, ${ }^{4}$ \\ Johann Helmut Brandstätter, ${ }^{1}$ and ${ }^{\circ}$ Hanna Regus-Leidig ${ }^{1}$ \\ ${ }^{1}$ Department of Biology, Animal Physiology, Friedrich Alexander University Erlangen-Nürnberg, 91058 Erlangen, Germany, ${ }^{2}$ Department of \\ Ophthalmology, University Hospital Erlangen, 91058 Erlangen, Germany, ${ }^{3}$ Synaptic Systems GmbH, 37079 Göttingen, Germany, and ${ }^{4}$ Institute of \\ Neuropathology and Department of Epileptology, University of Bonn, 53111 Bonn, Germany
}

RAB3A-interacting molecule (RIM) proteins are important regulators of transmitter release from active zones. At conventional chemical synapses, RIMs contribute substantially to vesicle priming and docking and their loss reduces the readily releasable pool of synaptic vesicles by up to $75 \%$. The priming function of RIMs is mediated via the formation of a tripartite complex with Munc13 and RAB3A, which brings synaptic vesicles in close proximity to $\mathrm{Ca}^{2+}$ channels and the fusion site and activates Munc13. We reported previously that, at mouse photoreceptor ribbon synapses, vesicle priming is Munc13 independent. In this study, we examined RIM expression, distribution, and function at male and female mouse photoreceptor ribbon synapses. We provide evidence that RIM1 $\alpha$ and RIM1 $\beta$ are highly likely absent from mouse photoreceptors and that RIM $2 \alpha$ is the major large RIM isoform present at photoreceptor ribbon synapses. We show that mouse photoreceptors predominantly express RIM2 variants that lack the interaction domain for Munc13. Loss of full-length RIM2 $\alpha$ in a RIM $\alpha \alpha$ mutant mouse only marginally perturbs photoreceptor synaptic transmission. Our findings therefore strongly argue for a priming mechanism at the photoreceptor ribbon synapse that is independent of the formation of a RIM-Munc13-RAB3A complex and thus provide further evidence for a fundamental difference between photoreceptor ribbon synapses and conventional chemical synapses in synaptic vesicle exocytosis.

Key words: active zone; Munc13; Piccolo; retina; vesicle priming; vesicle tethering

Significance Statement

RAB3A-interacting molecules 1 and 2 (RIM1/2) are essential regulators of exocytosis. At conventional chemical synapses, their function involves $\mathrm{Ca}^{2+}$ channel clustering and synaptic vesicle priming and docking through interactions with Munc13 and RAB3A, respectively. Examining wild-type and RIM2 mutant mice, we show here that the sensory photoreceptor ribbon synapses most likely lack RIM1 and predominantly express RIM2 variants that lack the interaction domain for Munc13. Our findings demonstrate that the photoreceptor-specific RIM variants are not essential for synaptic vesicle priming at photoreceptor ribbon synapses, which represents a fundamental difference between photoreceptor ribbon synapses and conventional chemical synapses with respect to synaptic vesicle priming mechanisms.

\section{Introduction}

RAB3A-interacting molecule (RIM) proteins are important regulators of synaptic vesicle exocytosis (Wang et al., 1997; Schoch et

\footnotetext{
Received Sept. 5, 2016; revised May 15, 2017; accepted June 18, 2017.

Author contributions: M.L. and H.R.-L. designed research; M.L., N.B., T.M., K.G., J.A., A.J., A.P., H.M., and H.R.-L. performed research; M.L., N.B., T.M., K.G., J.A., A.J., A.P., H.M., A.F., J.K., S.S., J.H.B., and H.R.-L. analyzed data; N.B., J.A., A.F., J.K., J.H.B., and H.R.-L. wrote the paper.

This work was supported by the Deutsche Forschungsgemeinschaft (Grant BR 1643/4-2 to H.R.-L. and J.H.B.). We thank F. Boggasch, K. Pertschy, and N. Gie $\beta$ I for excellent technical assistance; A. Gie $\beta$ I and Pieger for help with cloning; and F. Horn for help with statistics.

The authors declare no competing financial interests.

Correspondence should be addressed to Dr. Hanna Regus-Leidig, FAU Erlangen-Nürnberg, Department of

Biology, Animal Physiology, Staudtstr. 5, 91058 Erlangen, Germany. E-mail: hanna.regus-leidig@fau.de.
}

al., 2002; Calakos et al., 2004). The vertebrate RIM protein family is encoded by four genes and consists of seven members $(\mathrm{RIM} 1 \alpha / \beta, \operatorname{RIM} 2 \alpha / \beta / \gamma, \operatorname{RIM} 3 \gamma$, and RIM4 $\gamma$ ), which contain a set of conserved protein interaction domains and are capable of interacting with a variety of other synaptic proteins (Wang and Südhof, 2003; Mittelstaedt et al., 2010). One important role of RIMs is the regulation of $\mathrm{Ca}^{2+}$ channel clustering and function (Han et al., 2011; Kaeser et al., 2011; Grabner et al., 2015; Jung et al., 2015). Moreover, $\alpha$-RIMs coordinate key functions in neurotransmitter release; that is, vesicle docking presumably via in- 
teraction with the vesicle associated protein RAB3A and vesicle priming through activation of the priming factor Munc13, resulting in the assembly of the trans-SNARE complex (Wang et al., 1997; Betz et al., 2001; Kaeser et al., 2008; Deng et al., 2011; Ma et al., 2011; Südhof, 2013; Yang et al., 2015). In the absence of $\alpha$-RIMs, most chemical synapses exhibit a severe reduction in the readily releasable vesicle pool and impaired transmission (Schoch et al., 2002, 2006; Calakos et al., 2004).

At the tonically releasing photoreceptor ribbon synapses, the presence of the two large RIM1 and RIM2 isoforms (tom Dieck et al., 2005) and two short RIM3 $\gamma$ and RIM4 $\gamma$ isoforms (AlvarezBaron et al., 2013) has been reported. A distinctive localization of RIM1 and RIM2 at the photoreceptor ribbon synaptic compartment and the arciform density compartment was suggested, leading to the hypothesis that RIM1 and RIM2 may fulfill separate functions at the photoreceptor ribbon synapse (tom Dieck et al., 2005; Tian et al., 2012; Hallermann and Silver, 2013). However, their functions remained elusive and only recently was it shown that the absence of both RIM1 and RIM2 reduces $\mathrm{Ca}^{2+}$ influx into rod photoreceptor terminals through direct or indirect modulation of the $\mathrm{Ca}_{\mathrm{v}} 1.4$ channel, whereas $\mathrm{Ca}^{2+}$ channel expression and clustering was not impaired in the RIM1/2 double knock-out (Grabner et al., 2015). The latter study did not discriminate between a potentially different contribution of RIM1 and RIM2 to $\mathrm{Ca}^{2+}$ channel function.

In the present study, we reexamined the expression and localization of RIM1 and RIM2 at mouse photoreceptor ribbon synapses. We show that RIM $2 \alpha$ is the prevalent large RIM isoform present at mouse photoreceptor ribbon synapses and that the previously reported association of RIM1 with the synaptic ribbon compartment (tom Dieck et al., 2005; Regus-Leidig et al., 2009, 2010) may be explained by cross-reactivity of the used anti-RIM1 antibody with the ribbon-associated protein Piccolino (RegusLeidig et al., 2013). We characterized the photoreceptor RIM2 $\alpha$ in more detail and analyzed the importance of the RIMMunc13-RAB3A interaction for photoreceptor ribbon synaptic transmission. Our results strongly support an unconventional, RIM- and Munc13-independent, synaptic vesicle priming mechanism at the photoreceptor ribbon synapses.

\section{Materials and Methods}

Ethics statement. All experiments were performed in compliance with the guidelines for the welfare of experimental animals issued by the Federal Government of Germany, and the University of Erlangen-Nürnberg.

Animals. Adult (2-3 months of age) male and female C57BL/6 mice, RIM1 $\alpha$ mutant mice (RIM $1 \alpha^{\mathrm{mt}}$; Schoch et al., 2002), RIM2 $\alpha$ mutant mice (RIM $2 \alpha^{\mathrm{mt}}$; Schoch et al., 2006), and RIM $2 \alpha /$ Rac3-eGFP mice, generated by crossing the RIM $2 \alpha^{\mathrm{mt}}$ mice with $\mathrm{Tg}($ Rac3-eGFP)JZ58Gsat/ Mmcd (Rac3-eGFP) mice, were used in the study. Rac3-eGFP mice expressing eGFP in cone photoreceptors (Regus-Leidig et al., 2013) were obtained from the Mutant Mouse Regional Resource Center (MMRRC), a National Center for Research Resources (NCRR)-National Institutes of Health (NIH)-funded strain repository, and were donated to the MMRRC by the National Institute of Neurological Disorders and Stroke (NINDS)-funded Gene Expression Nervous System Atlas (GENSAT) bacterial artificial chromosome (BAC) transgenic project. Mice were kept in a $12 \mathrm{~h} / 12 \mathrm{~h}$ light/dark cycle with lights on at 6:00 A.M.

Fluorescence-activated cell sorting (FACS) and laser microdissection. For sorting of eGFP-positive cone photoreceptors, RIM $2 \alpha^{\text {wt }} /$ Rac3-eGFP retinae were dissociated using $20 \mathrm{U} / \mathrm{ml}$ papain and resuspended in FACS buffer (2\% FCS, 2 mm EDTA in $0.1 \mathrm{~m}$ PBS, pH 7.4). Cells were sorted with a FACSAria III Cell Sorter (BD Biosciences) and collected in RLT buffer (Qiagen) containing 1\% $\beta$-mercaptoethanol. Outer nuclear layer samples were collected from $70 \mu \mathrm{m}$ vertical cryostat sections by laser microdissection. The sections were collected on membrane covered slides
(FrameSlide $1.4 \mu \mathrm{m}$ PET; Leica), fixed for $2 \mathrm{~min}$ in $-20^{\circ} \mathrm{C}$ cooled $70 \%$ ethanol, and washed in ultra-pure water at room temperature. Sections were stained with $1 \%$ cresyl violet in $50 \%$ ethanol for $20 \mathrm{~s}$ at room temperature and finally washed in $70 \%$ ethanol at room temperature. Dried sections were dissected immediately with the Leica LMD7 system equipped with a UV laser $(349 \mathrm{~nm})$. The dissected samples were collected in RLT buffer (Qiagen) containing 1\% $\beta$-mercaptoethanol.

RNA isolation and RT-PCR. Total RNA was isolated using the RNeasy Mini Kit (whole tissue) or the RNeasy Micro Kit (sorted cells, microdissected tissue) (Qiagen). Isolated RNA was reverse transcribed using random hexamers, M-MLV reverse transcriptase, $5 \times$ RT buffer, a mixture of dNTPs, Rnasin (Promega), and $1 \mu \mathrm{g}$ of total RNA (whole retina and cortex), $0.5 \mu \mathrm{g}$ of total RNA (organ of Corti), complete RNA sample (microdissected tissue), and the complete RNA sample from 20,000 cones in a final volume of $25 \mu \mathrm{l}$. For the PCR, $1 \mu \mathrm{l}$ (retina and cortex), $1.5 \mu \mathrm{l}$ (organ of Corti), or $2 \mu \mathrm{l}$ (microdissected tissue, sorted cells) of cDNA were amplified in a final volume of $25 \mu \mathrm{l}$ with $0.75 \mathrm{U}$ of TaqDNA polymerase (Qiagen) and 10 pmol of each primer. Cycling conditions were as follows: 35 cycles at $94^{\circ} \mathrm{C}$ for $30 \mathrm{~s}, 59^{\circ} \mathrm{C}$ for $45 \mathrm{~s}$, and $72^{\circ} \mathrm{C}$ for 1 min followed by a final $72^{\circ} \mathrm{C}$ extension step for $10 \mathrm{~min}$. Amplicon sizes were determined on $1.5 \%$ agarose gels stained with $\mathrm{EtBr}$ (Roth) and photographed using a computer-assisted gel documentation system (DeVision G; Decon Science). Negative controls were treated as above without adding template. The identity of the PCR products was verified by DNA sequencing (Eurofins Genomics). Nucleotide alignments were generated with CLC Sequence Viewer 6.

The following primers specific for RIM $1 \alpha / \beta$ (Rims1-204: ENSMUST00000097811; RIM1 $\beta$ Exon 1: GenBank: FJ472653.1), RIM $2 \alpha / \beta$ (RIM2-202: ENSMUST00000082054), RIM3 $\gamma$ (Rims3-001: ENSMUST00000071093), RIM4 $\gamma$ (Rims4-001: ENSMUST00000044734), $\beta$-Actin (Actb-001: ENSMUST00000100497), and Complexin2 (Cplx2001: ENSMUST00000026985) were used for RT-PCR and sequencing:

RIM1 $\alpha$ forward primer (F): 5' -CTCCCCCTATGCAAGAACTG-3'; RIM1 $\beta$ F: $5^{\prime}$-CTGTCCCATTTTCCTCTCCA-3'; RIM $1 \alpha / \beta$ reverse primer (R): 5'-AGGACCACTTCCGAAGAACC-3'; RIM $2 \alpha$ F: 5'-GCT GTCATGGATCGTCAGAA- $3^{\prime}$; RIM $2 \beta$ F: $5^{\prime}$-TCTCATTTCCATGGGGT TTT-3'; RIM $2 \alpha / \beta$ R: 5'-CTTTGGAATGTCTATGGCCTC-3'; RIM $2 \alpha$ Ex1/6 F: 5'-CCGTGCTCAAAAAACGAAGT-3'; RIM3 $\gamma$ F: 5'-GGAGCACAG AGACTGGCATT-3'; RIM3 $\gamma$ R: 5' -CCATACCCATGAAGCACTTG-3'; RIM4 $\gamma$ F: 5'-TGACTTCCTGGGGAGTATGG-3'; RIM4 $\gamma$ R: 5'-GGT CAAGTCCAGTTCCTCCA-3'; $\beta$-Actin (F: $5^{\prime}$-TTCCTCCCTGGAGAA GAG-3'; R:5'-CACTGTGTTGGCATAGAG-3'); Complexin2(F:5'-AGT GGCTTAGACGGTTG-3'; R: 5' -TGCAGGCTTTGGTTAATG-3').

DNA constructs and cell culture. Generation of the RIM1 $\alpha$ expression vector was described previously (Lonart et al., 2003); RIM $2 \alpha$ and RIM $2 \beta$ expression vectors were a kind gift from Thomas Südhof (Kaeser et al., 2012). Fragments of Piccolo (aa1-241; Pclo-201: ENSMUST00000030691), ubMunc13-2 (aa 1-315; Unc13b-012: ENSMUST00000107952), RIM2 $\alpha$ $\left(\mathrm{RIM} 2 \alpha^{\Delta \mathrm{EX} 2 / 3}\right.$; aa $16-444$ without aa $60-99 ; \mathrm{RIM} 2 \alpha^{\Delta \mathrm{Ex} 2-5}$; aa $16-444$ without aa 60-273), and full-length RAB3A (aa 1-220; RAB3A-001: ENSMUST00000034301.11) were amplified from C57BL/6JRj mouse retinal cDNA by PCR and ligated into pCR8/GW/TOPO vector (Life Technologies). The eYFP-tagged fragment of Pclo-aa 1-241 was generated by subcloning into the destination vector eYFP/Dest N-Term Erl.5.6 via LR reaction (Life Technologies); the ubMunc13-2 and RAB3A fragments were subcloned into the destination vector pcDNA3native_Dest and the RIM $2 \alpha$ fragments into the destination vector pDest 27 (Life Technologies).

HEK293T and NIH 3T3 cells were transfected with TransIT-Pro transfection reagent (Mirus) according to the manufacturer's recommendations or with polyethylenimin (Polysciences).

GST pulldown. Supernatants of HEK293T lysates expressing the GST fusion proteins were diluted 1:1 in HNTG buffer (10\% glycerol, $20 \mathrm{~mm}$ HEPES, $120 \mathrm{~mm} \mathrm{NaCl}, 0.3 \%$ Triton X-100, pH 7.3) before loading onto Glutathione Sepharose 4B beads (GE Healthcare). After a $2 \mathrm{~h}$ incubation step in an overhead shaker, the beads were washed three times in HNTG buffer. Subsequently, cell lysates containing the prey protein were added to the beads and incubated overnight in an overhead shaker. $1 \mu \mathrm{M}$ final concentration of GTP $\gamma \mathrm{S}$ (Sigma-Aldrich) was added to keep RAB3A in 
the active GTP-bound state. Pulldowns were washed 3 times in HNTG buffer before elution in SDS-sample buffer (10\% glycerol, $250 \mathrm{~mm}$ Tris, 0.5 mm EDTA, 2\% SDS, 0.001\% bromophenol blue, 195 mm dithiothreitol, DTT) at $95^{\circ} \mathrm{C}$ followed by SDS gel electrophoresis and Western blotting.

Western blot analysis. For the antibody specificity test, transfected cells were lysed in lysis buffer ( $50 \mathrm{~mm}$ Tris-HCl pH 7.5, 5 mm EDTA, $150 \mathrm{~mm}$ $\mathrm{NaCl}, 1 \%$ Triton X-100) and samples were sonicated and centrifuged at $20,000 \times g$ for $15 \mathrm{~min}$. The supernatants were diluted in $5 \times$ Lämmlibuffer (50\% glycerol, 5 mм EDTA, 4\% SDS, 250 mм DTT, $0.1 \%$ bromophenol blue). Proteins were separated by SDS-PAGE using $10 \%$ polyacrylamide gels and transferred to PVDF membranes (Immobilon-P; Merck/Millipore) by semidry blotting (Trans Blot Turbo; Bio-Rad).

Retinal tissue was homogenized in lysis buffer (320 mm saccharose, $4 \mathrm{~mm}$ HEPES, $\mathrm{pH} 7.5)$ and centrifuged at $1000 \times \mathrm{g}$ for $10 \mathrm{~min}$. The supernatant (S1) was centrifuged at $20,000 \times g$ for 20 min. Pellets (P2) were dissolved in SDS-sample buffer (10\% glycerol, $250 \mathrm{~mm}$ Tris, $0.5 \mathrm{~mm}$ EDTA, 2\% SDS, 0.001\% bromophenol blue, 195 mм DTT). Proteins were separated by SDS-PAGE using $5 \%$ polyacrylamide gels ( $20 \mu \mathrm{g} / \mathrm{lane})$ and transferred to PVDF membranes by tank blotting (Trans-Blot Cell; Bio-Rad).

For immunodetection, membranes were blocked in blocking solution (10 mм Tris, $150 \mathrm{~mm} \mathrm{NaCl,} \mathrm{0.2 \%} \mathrm{blocking} \mathrm{reagent;} \mathrm{AppliChem)} \mathrm{or} \mathrm{skim}$ milk powder and primary antibodies were applied overnight at $4^{\circ} \mathrm{C}$. HRP-conjugated secondary antibodies were visualized by chemiluminescent detection (Luminata Forte; Millipore). Images were obtained with a molecular imager (ChemiDoc XRS; Bio-Rad) and adjusted for contrast and brightness using Adobe Photoshop CS.

Antibodies. The following primary antibodies were used for immunocytochemistry (ICC) and Western blotting (WB): monoclonal mouse anti-CtBP2/RIBEYE (ICC 1:10,000; BD Biosciences), mouse antiRAB3A (WB 1:5000; Synaptic Systems), polyclonal goat anti-GST (WB 1: 10,000; GE Healthcare), polyclonal rabbit anti-Pclo 4 (ICC 1:1000; WB 1:4000; Regus-Leidig et al., 2013), rabbit anti-RIM1ab1 (ICC/WB 1:1000-1:2000; \#140 003; Synaptic Systems), rabbit anti-RIM1ab2 (ICC/WB 1:1000-1:2000; \#140 013; Synaptic Systems), rabbit antiRIM1ab3 (ICC/WB 1:1000-1:2000; \#140 023; Synaptic Systems), rabbit anti-RIM2ab1 (ICC/WB 1:1000-1:2000; \#140 103; Synaptic Systems), rabbit anti-RIM2ab2 (ICC/WB 1:1000-1:2000; \#364 003; Synaptic Systems), rabbit anti-RIM2ab2 depl (ICC/WB 1:1000-1:2000; \#140 203; Synaptic Systems), rabbit anti-RIM2ab3 (ICC/WB 1:1000-1:2000; \#140 303; Synaptic Systems), and rabbit anti-ubMunc13-2 (WB 1:1000; Cooper et al., 2012). Nuclei were stained with 4,6-diamidino-2-phenylindole (DAPI; 1:50,000; Sigma-Aldrich).

The antibody RIM2ab2 ${ }^{\text {depl }}$ was obtained as follows: $1 \mathrm{mg}$ of synthetic peptide (CDLSQLSEEERRQIAAVMSR) corresponding to aa 41-59 of mouse Piccolo (Pclo-201: ENSMUST00000030691) were coupled to SulfoLink agarose (Thermo Fisher) according to the manufacturer's instructions. Then, $5 \mathrm{ml}$ of rabbit serum raised against recombinant aa 1-466 of rat Rim2 (RIM2ab2) was passed over this column to remove antibodies cross-reactive to this epitope. Bound antibodies were eluted and collected for further characterization. The flow-through was further affinity purified with recombinant rat Rim2 (aa 1-466) coupled to cyanogen bromide-activated Sepharose 4B (GE Healthcare). This antibody is hereafter referred to as RIM2ab2 ${ }^{\mathrm{depl}}$.

The following secondary antibodies were used: Alexa Fluor 488/594conjugated goat anti-mouse and goat anti-rabbit IgG (ICC 1:500; WM 1:250; Invitrogen), Cy3/Cy5-conjugated goat anti-mouse and goat antirabbit IgG (1:100-1:200; Dianova; Jackson Immunoresearch), and HRPconjugated goat anti-mouse/rabbit IgG (1:10,000; Sigma-Aldrich).

Tissue preparation and light microscopic immunocytochemistry. Preparation of retinal tissue and antibody incubation for light microscopic immunocytochemistry were done as described previously (Dick et al., 2001). Briefly, the eyes were opened and retinae were immersion fixed in the eyecup for $15 \mathrm{~min}$ in $4 \%$ paraformaldehyde in phosphate buffer (PB; $0.1 \mathrm{M}, \mathrm{pH} 7.4$ ). The retinae were mounted in freezing medium (ReichertJung) and $12-\mu \mathrm{m}$-thick horizontal sections were cut with a cryostat (Leica CM3050 S). Primary antibody incubation was performed over- night at room temperature, followed by secondary antibody incubation for $1 \mathrm{~h}$. Labeled sections were examined with a Zeiss Axio Imager Z1 equipped with an ApoTome or a Zeiss confocal laser scanning microscope LSM 710 . Images were adjusted for contrast and brightness using Adobe Photoshop CS.

For comparison of RIM $2 \alpha^{\mathrm{mt}}$ and RIM $2 \alpha^{\mathrm{wt}}$ stainings, retinal slices of the two genotypes were processed in parallel and images were acquired using the same camera, laser, and photo-multiplier tube settings. For the comparative fluorescence intensity analysis, $3 \mathrm{D}$ reconstructions from confocal $z$-stacks were generated with Imaris software (Bitplane) using the same threshold for reconstruction of single synaptic ribbons.

For immunocytochemical stainings of transfected cells, the cells were fixed $24 \mathrm{~h}$ after transfection for $15-20 \mathrm{~min}$ in $4 \%$ PFA in PB $(0.1 \mathrm{M}, \mathrm{pH}$ 7.4) and subjected to immunocytochemistry as described above.

Electrophysiology. Preparation of retinal slices and experiments were performed under dim red light. For vertical slices, adult mice (1-3 months old) were anesthetized with isoflurane and killed by cervical dislocation. After enucleation and isolation of the retina, the tissue was cut into several large pieces. Each retinal piece was placed vitreal side down on a filter paper $(0.8 \mu \mathrm{m}$ pores; Millipore). Vertical retinal slices of $125 \mu \mathrm{m}$ thickness were cut in saline solution with a razor blade tissue chopper (Stoelting) and positioned in the recording chamber on an upright fixed stage microscope and observed with a 63/1.0 W Plan Apochromat objective (Examiner D1; Zeiss filter set $38 \mathrm{HE}$ and 63HE). Slices were kept at room temperature in a bicarbonate-buffered extracellular solution containing the following (in mM): $119 \mathrm{NaCl}, 25 \mathrm{NaHCO}_{3}, 1.25$ $\mathrm{NaH}_{2} \mathrm{CO}_{3}, 2.5 \mathrm{KCl}, 2 \mathrm{CaCl}_{2}, 1 \mathrm{MgCl}_{2}, 10$ glucose, $2 \mathrm{Na}$-pyruvate, and 0.4 ascorbic acid, $\mathrm{pH} 7.2$, when oxygenated with $95 \% \mathrm{O}_{2} / 5 \% \mathrm{CO}_{2}$. The preparation of horizontal slices was described in detail in a previous study (Feigenspan and Babai, 2017). Briefly, after isolation of the retina, the tissue was cut into $4-6$ pieces and embedded in $1.8 \%$ low gelling agarose (Sigma-Aldrich). Horizontal slices of 160-200 $\mu \mathrm{m}$ thickness were cut with a vibratome (Leica Microsystems) at room temperature. During recordings, retinal slices were perfused with oxygenated extracellular solution at a speed of $\sim 1 \mathrm{ml} / \mathrm{min}$.

Whole-cell recordings were obtained using $8-15 \mathrm{M} \Omega$ patch electrodes fabricated from borosilicate glass $(1.5 \mathrm{~mm}$ outer diameter, $0.86 \mathrm{~mm}$ inner diameter; Sutter Instruments) on a P-97 micropipette puller (Sutter Instruments). The pipette solution contained the following (in $\mathrm{mM}$ ): 136.6 cesium gluconate, 13 TEACl, 4 MgATP, $0.4 \mathrm{NaGTP}, 5$ EGTA, and 15 HEPES, pH 7.2. Patch-clamp recordings were performed with an EPC-10 patch-clamp amplifier (HEKA) controlled by the Patchmaster software package (HEKA). Currents through voltage-gated calcium channels were obtained using a ramp voltage protocol $(-60$ to $+50 \mathrm{mV}$, $0.11 \mathrm{mV} / \mathrm{ms}$ ) applied from a steady holding potential of $-60 \mathrm{mV}$. Passive membrane resistances were measured between -60 and $-50 \mathrm{mV}$ and subtracted post hoc.

Mouse cone photoreceptors were identified by using specific mouse line in which all of the cones were fluorescently labeled with green fluorescent protein (RIM2 $\alpha /$ Rac3-eGFP). In some cases, $100 \mu \mathrm{M}$ Alexa Fluor 568 (Life Technologies) dissolved in intracellular solution was injected to visualize cone photoreceptors during or after whole-cell recording (see Fig. $7 H$ ). Photoreceptors were stimulated with attenuated full-field flashes of $130 \mathrm{~W} / \mathrm{cm}^{2}$ band-pass-filtered light $(470 \pm 40 \mathrm{~nm}$; corresponding to $3.075 \times 10^{12}$ photons $/ \mathrm{s} / \mu \mathrm{m}^{2}$ ) using the xenon arc lamp of the Lambda DG 4 illumination system (Sutter Instruments). The relatively dark-adapted condition refers to sustained exposure to dim red light $\left(620-645 \mathrm{~nm}, \sim 1 \mathrm{~mW} / \mathrm{m}^{2}\right)$. Rise time values of light responses refer to the $20-80 \%$ rising phase of the light evoked outward current.

Current traces were analyzed with Fitmaster, Patchmaster (both HEKA) and IgorPro 6.2 (WaveMetrics) software. The region fitted with the Boltzmann function extended from baseline to just beyond the maximum $\mathrm{Ca}^{2+}$ current to avoid influences from outward $\mathrm{K}^{+}$currents activated at more positive membrane potentials. Parameters obtained from the fit were peak amplitude, half-maximal activation $\left(V_{50}\right)$, and slope $(k)$ of the current. All data are reported as mean \pm SEM. For illustration purposes, current traces were low-pass filtered at $1 \mathrm{kHz}$.

Electroretinography (ERG). The detailed procedure of measuring the ERG in mice has been described previously (Harazny et al., 2009). Briefly, 
the animals were dark adapted overnight and all further handling was performed under deep red illumination. The mice were anesthetized by an intramuscular injection of $50 \mathrm{mg} / \mathrm{kg}$ ketamine (Ketavet; Pfizer) and $10 \mathrm{mg} / \mathrm{kg}$ xylazine (Rompun 2\%; Bayer). A subcutaneous injection of saline solution $(10 \mathrm{ml} / \mathrm{kg}, 0.9 \%)$ was administered to prevent desiccation. The pupils were dilated with a drop of tropicamide (Mydriaticum Stulln, $5 \mathrm{mg} / \mathrm{ml}$; Pharma Stulln) and phenylephrine hydrochloride (Neosynephrin POS 5\%; Ursapharm). To measure the ERG, the active contact lens electrodes (Mayo) internally covered with Corneregel (Dr. Mann Pharma) were placed on the cornea of each eye. A gold needle electrode was placed subcutaneously at the base of the tail and served as ground. Reference electrodes were gold needles positioned subcutaneously next to the ipsilateral ears. To deliver the stimuli, a Ganzfeld Stimulator (Q450 SC; Roland Consult) was used. Stimulation and data recording were controlled using the RetiPort system (Roland Consult). Initially, the dark-adapted (scotopic) flash ERG was measured. The flash strength increased in eight steps $(0.0002,0.002,0.0063,0.02,0.063,0.2,0.63$, and $6.3 \mathrm{~cd} . \mathrm{s} / \mathrm{m}^{2}$ ) and, depending on flash strength, $8-12$ flashes were averaged. Interflash intervals varied between $5 \mathrm{~s}$ at the lowest flash strength to $14 \mathrm{~s}$ at $6.3 \mathrm{~cd} . \mathrm{s} / \mathrm{m}^{2}$. Flash duration varied between $5 \mu \mathrm{s}$ and $5 \mathrm{~ms}$. After the scotopic ERGs were recorded and before photopic flash ERG measurements, the animals were adapted to $25 \mathrm{~cd} / \mathrm{m}^{2}$ steady white background light for $5 \mathrm{~min}$. Photopic flash ERGs were obtained by delivering flash superimposed on the background. Five different strengths $(0.063,0.2$, $0.63,2$, and $6.3 \mathrm{~cd} . \mathrm{s} / \mathrm{m}^{2}$ ) were used. At each flash strength, 20 responses were averaged. Offline analyses of the responses were performed using custom-designed MATLAB (The MathWorks) routines and Excel (Microsoft) spreadsheets. From the scotopic flash ERG responses, the oscillatory potentials (OPs) were extracted by using a variable filter procedure as described by Harazny et al. (2009). Briefly, after Fourier transform of the ERG signal into the frequency domain, the OPs were identified as the signal in a frequency region above an amplitude minimum that was generally found between 40 and $70 \mathrm{~Hz}$. The amplitude of the OPs was defined as the amplitude maximum at frequencies between the minimum and $200 \mathrm{~Hz}$. The maximum typically occurred at frequencies between 80 and $120 \mathrm{~Hz}$. To obtain the conventional ERG without OPs, the amplitudes above the minimum were set to zero and the signal was inverse Fourier transformed. The phase plot was not altered by this procedure and the filter procedure cannot be compared with a hardware filter. The amplitudes and implicit times of the a- and b-waves were measured in the ERGs without OPs. The a-wave amplitude was defined as the difference between the baseline level before stimulus onset and the minimum of the a-wave. The b-wave amplitude was defined as the difference between the a-wave minimum and the b-wave maximum. Implicit times were defined as the time between stimulus onset and the minimum or maximum, respectively. The b-wave amplitude and implicit time of the photopic flash ERGs was measured in an analogous manner. Flicker ERGs were measured to sinusoidal stimulation around a mean luminance of $25 \mathrm{~cd} / \mathrm{m}^{2}$. Michelson contrast was $100 \%$. The measurements were repeated at four different temporal frequencies: 12, 18, 24 , and $30 \mathrm{~Hz}$. The response amplitudes and phases of the fundamental components after Fourier transform of the recorded responses were used.

Experimental design and statistical analysis. A total of $3 \operatorname{RIM} 1 \alpha^{\mathrm{wt}}, 3$ RIM $1 \alpha^{\mathrm{mt}}, 3$ RIM $2 \alpha^{\mathrm{wt}}$, and 4 RIM $2 \alpha^{\mathrm{mt}}$ mice was used for patch-clamp recordings. The age of the animals ranged from 8 to 16 weeks and males and females were represented in equal proportions. For each experiment, nine to 13 different cells from at least three mice of each genotype were recorded. All values obtained were tested for statistical significance. The comparison of means for two independent distributions was performed with the two-sample $t$ test, given the data were normally distributed. Normal distribution was tested with Shapiro-Wilk, whereas Levene's test was used for detecting differences in variances. Welch correction of the $t$ test was performed in those cases in which two groups displayed significantly different variances. Mann-Whitney $U$ test was used for comparison of data that could not be described by a normal distribution. Exact $p$-values, test statistics of experiments, and the number of animals and/or neurons are given in the respective figure legends. In all cases, statistical significance was accepted at the $p<0.05$ level.
For the ERG recordings, statistical differences between RIM $1 \alpha^{\mathrm{mt}} /$ RIM $2 \alpha^{\mathrm{mt}}$ mice (RIM1 $\alpha^{\mathrm{mt}}: n=6$, both sexes; RIM $2 \alpha^{\mathrm{mt}}: n=6$, both sexes) and age-matched wild-type mice (RIM1 $\alpha^{\mathrm{wt}}: n=4$, both sexes; RIM $2 \alpha^{\text {wt }}: n=5$, both sexes were used as one control group) were tested using unpaired $t$ test or Mann-Whitney $U$ test (SPSS version 21; IBM). Both eyes of each animal were used for evaluation. An $\alpha$-value of $p<0.05$ was adopted as the threshold for significance, but, due to multiple testing, the significance level was Bonferroni corrected for the analysis of each protocol (scotopic flash ERG: $\alpha_{\text {Bonferroni }}=0.0063$; photopic flash ERG: $\alpha_{\text {Bonferroni }}=0.01$; photopic flicker ERG: $\alpha_{\text {Bonferroni }}=0.025$ ).

All other experiments that were not statistically analyzed were independently repeated three or more times to ensure consistency and reproducibility.

\section{Results}

\section{RIM2 $\alpha$ is the prevalent large RIM isoform present at mouse photoreceptor ribbon synapses}

In a first set of experiments, we reexamined the expression and distribution of RIM1 and RIM2 in the mouse retina with RT-PCR and immunocytochemistry (Fig. 1). RT-PCR was performed with specific primer pairs for the different RIM isoforms and with cDNA from laser microdissected outer nuclear layer (ONL) and FACS-sorted cone photoreceptors (Fig. 1A). Samples from cortex and organ of Corti served as positive controls (Jung et al., 2015). Complexin2 (Cplx2), which is not present in photoreceptors (Reim et al., 2005), was used as a negative control to judge the purity of the photoreceptor samples for RT-PCR. Despite a weak contamination with Cplx2-expressing cells, the only consistently detectable large RIM isoform in the photoreceptor samples (ONL, cones) was RIM $2 \alpha$ (Fig. $1 A$ ). We also detected RIM3 $\gamma$ in the ONL and cone sample and a weak RIM4 $\gamma$ band in the ONL sample (Fig. 1A). These findings agree with previously published immunocytochemical data showing the presence of the short RIM3 $\gamma$ and RIM4 $\gamma$ isoforms in photoreceptor terminals (AlvarezBaron et al., 2013). For the immunocytochemical experiments, six antibodies directed against different epitopes of RIM1 and RIM2 were used (Fig. $1 B$ ). We tested antibody specificity on Western blots of overexpressed RIM1 $\alpha$, RIM $2 \alpha$, and RIM2 $\beta$ (Fig. $1 B$, right). Only RIM1ab2 and RIM1ab3 were isoform specific. Of the tested RIM2 antibodies, RIM2ab3 was the antibody with the highest RIM2 specificity because it stained RIM1 only weakly (Fig. 1B). Immunocytochemical stainings of RIM1 in vertical cryostat sections of C57BL/6 mouse retina with the two RIM1specific antibodies (RIM1ab2 and RIM1ab3; Fig. 1C) support an absence of RIM1 protein from photoreceptors and their ribbon synapses in the outer plexiform layer (OPL). Because the two RIM1-specific antibodies do not show any staining in the OPL, we interpret the arciform density staining in rod photoreceptors with RIM1ab3 to result from cross-reactivity with RIM2 (Fig. 1C). In contrast to RIM1, RIM2 (RIM2ab1 and RIM2ab3) staining was present at photoreceptor ribbon synapses and doublelabeling experiments with the synaptic ribbon marker RIBEYE demonstrated its localization at the arciform density in rod photoreceptor synapses (Fig. 1C). This finding agrees well with previously published data (tom Dieck et al., 2005; Regus-Leidig et al., 2010).

The antibody RIM2ab2 produced a staining that overlapped with the RIBEYE staining in rod photoreceptor synapses (Fig. $1 C)$. Because RIM2 had never before been reported at synaptic ribbons and RIM1/2 $\alpha$ and Piccolo share an 18 aa homology region in their first exon (Fig. $2 A$ ), we tested for a possible crossreactivity of RIM2ab2 with the synaptic ribbon-associated Piccolo variant Piccolino (Wang et al., 2001; Regus-Leidig et al., 2013). Piccolo aa1-241 N-terminally fused to eYFP (eYFP-Pclo- 
A

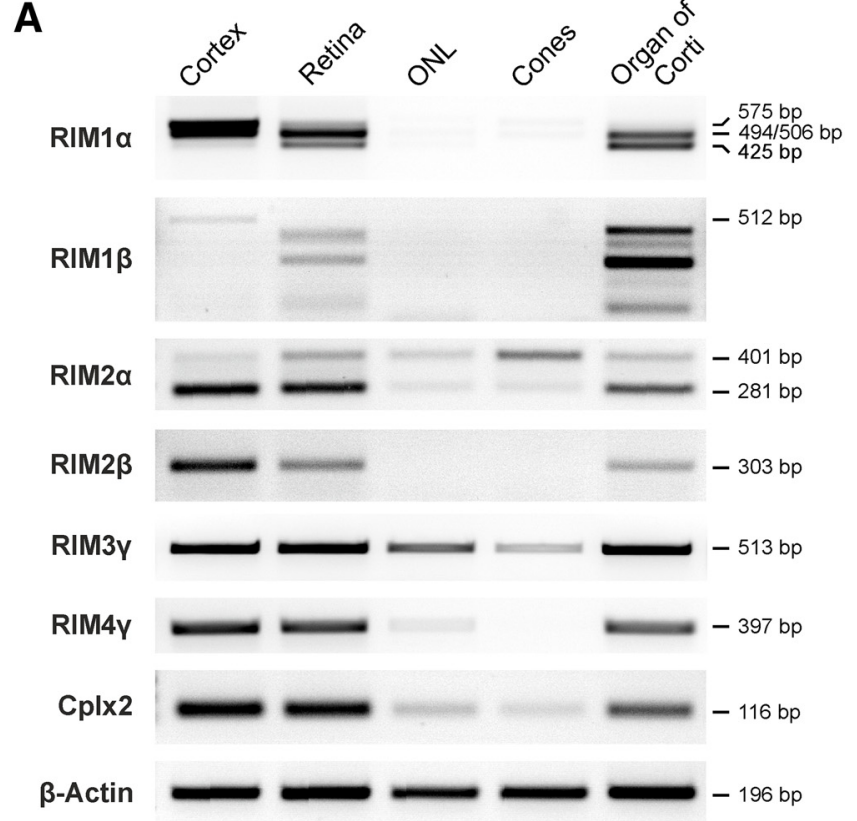

B
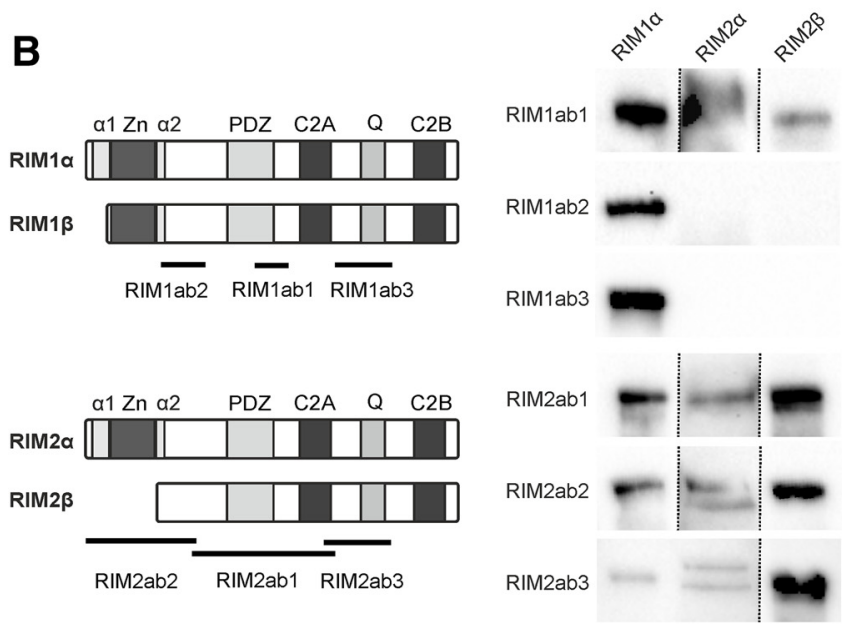

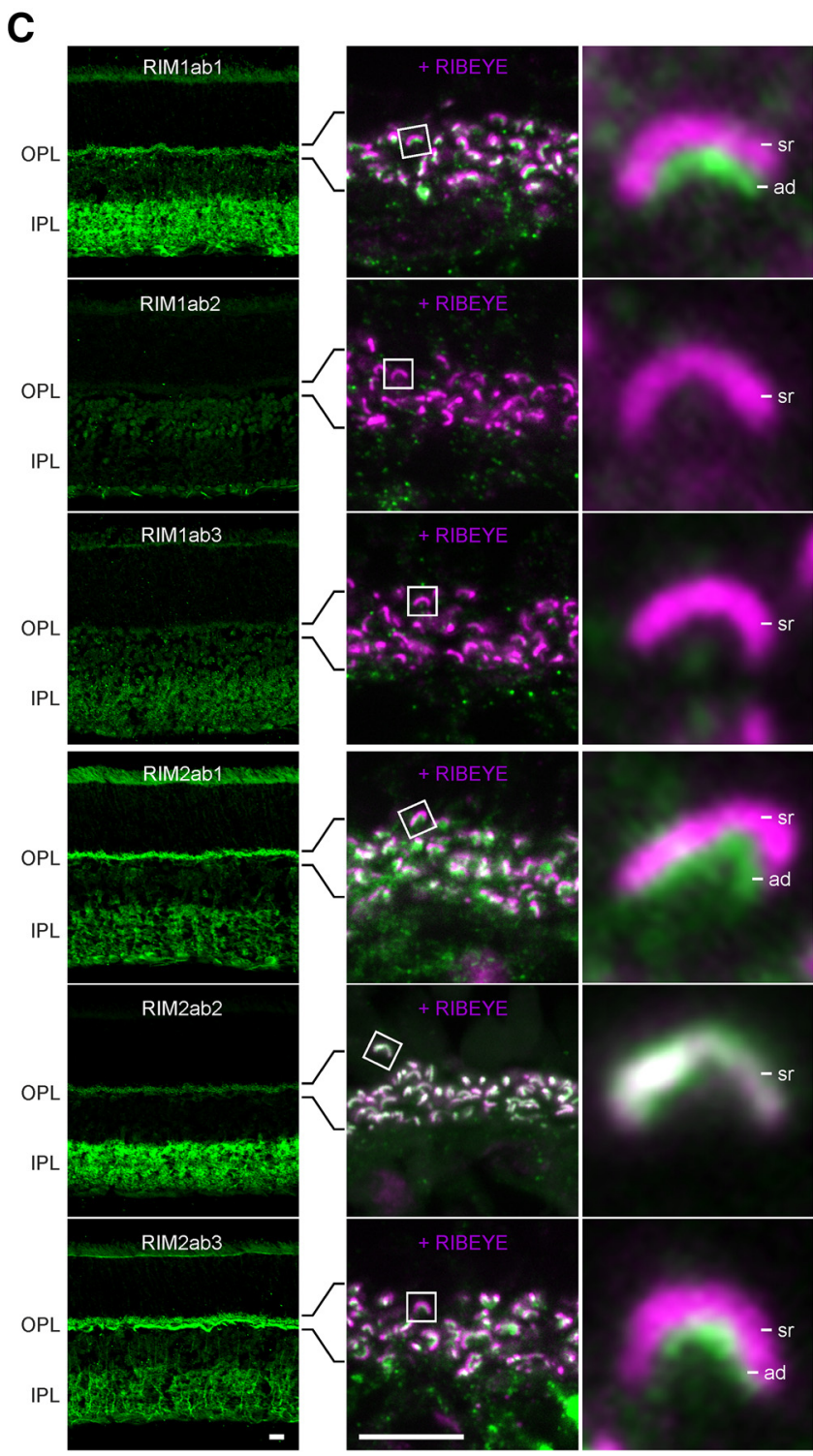

Figure 1. Expression and distribution of RIM family members at photoreceptor ribbon synapses. $A$, RT-PCR of CDNA from cortex, whole retina, ONL, isolated cone photoreceptors (Cones), and organ of Corti with specific primers for the different RIM isoforms. As a control for the purity of the photoreceptor samples, primers for Complexin2 (Cplx2) were used and RT-PCR with $\beta$-Actin primers served as loading control. B, Left, Schematic representation of RIM $1 \alpha / \beta$ and RIM $2 \alpha / \beta$ and epitope locations of the used antibodies. Right, Western blots of overexpressed RIM $1 \alpha$, RIM $2 \alpha$, and RIM2 $\beta$ stained with the different RIM antibodies to test for antibody specificity. C, Left, Images of vertical sections through the C57BL/6 mouse retina stained with the different RIM antibodies. Right, Immunocytochemical double staining of the OPL with the different RIM antibodies (green) and with an antibody against RIBEYE (magenta). $\alpha 1 / \alpha 2$, RAB3A-binding domains; ad, arciform density; bp, base pairs; C2, C2 domain; PDZ, PDZ domain; $Q$, glutamine-rich heptad repeat; sr, synaptic ribbon; Zn, zinc finger. Scale bar in C, $5 \mu \mathrm{m}$.

aa1-241) was expressed in HEK293T cells and labeled with the antibodies Pclo4 (directed against the $\mathrm{N}$ terminus of Piccolo/ Piccolino) and RIM2ab2 (Fig. 2B). eYFP-Pclo-aa1-241 (green) was diffusely distributed in transfected HEK293T cells including the nucleus and it was detected by both Pclo4 (Fig. 2B, top, red) and RIM2ab2 (Fig. 2B, bottom, red). On Western blots of wildtype mouse retina $\mathrm{P} 2$ fractions, RIM2ab2 detected in addition to RIM1 and RIM2 two high-molecular-weight bands $(>300 \mathrm{kDa})$. These two bands are of comparable size to Piccolo/Piccolino detected by Pclo4 (Fig. 2C). The cross-reactivity of RIM2ab2 was abolished by purification against the N-terminal RIM homology region of Piccolo/Piccolino (aa41-aa59: DLSQLSEEERRQIAAVMSR; RIM2ab2 ${ }^{\text {depl; }}$ Fig. $2 D, E$ ). Finally, immunocytochemical double labeling of the OPL with the purified RIM2ab2 ${ }^{\text {depl }}$ (green) and an antibody against RIBEYE (magenta) proved the absence of ribbon labeling in rod photoreceptor synapses (Fig. 1C,F). Instead, RIM2ab2 ${ }^{\text {depl }}$ labeled the rod photoreceptor arciform density and was now consistent with the RIM2ab1 and RIM2ab3 staining pattern (Fig. $2 F$ ).

The lack of RIM1 labeling at mouse photoreceptor ribbon synapses contradicts our previous findings (tom Dieck et al., 2005; Regus-Leidig et al., 2009, 2010). To analyze the contribution of RIM $1 \alpha$ to photoreceptor synaptic transmission, we measured ERGs from RIM1 $\alpha$-deficient mice (RIM1 $\alpha^{\mathrm{mt}}$; Fig. $3 A-F$; Schoch et al., 2002). The amplitudes of the a-waves, b-waves, and OPs and the a-wave delay of the scotopic flash ERG revealed no differences between wild-type and RIM1 $\alpha^{\mathrm{mt}}$ mice (Fig. $3 A-C$ and Fig. 3-1 available at https://doi.org/10.1523/JNEUROSCI. 2795-16.2017.f3-1) with the exception that the b-wave implicit time was slightly smaller in the RIM $1 \alpha^{\mathrm{mt}}$ mice at medium flash intensities (Fig. 3B and Fig. 3-1 available at https://doi.org/10. 1523/JNEUROSCI.2795-16.2017.f3-1). The photopic flash ERG 
A

40

60

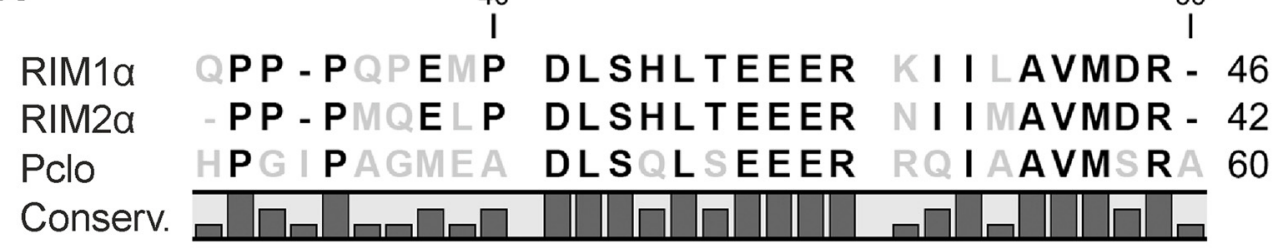

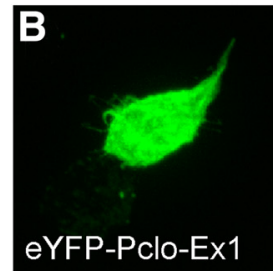

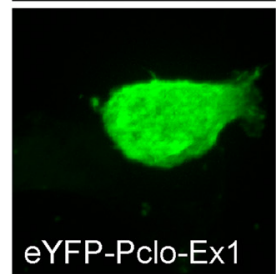

D
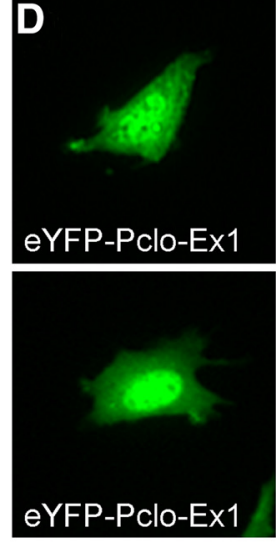
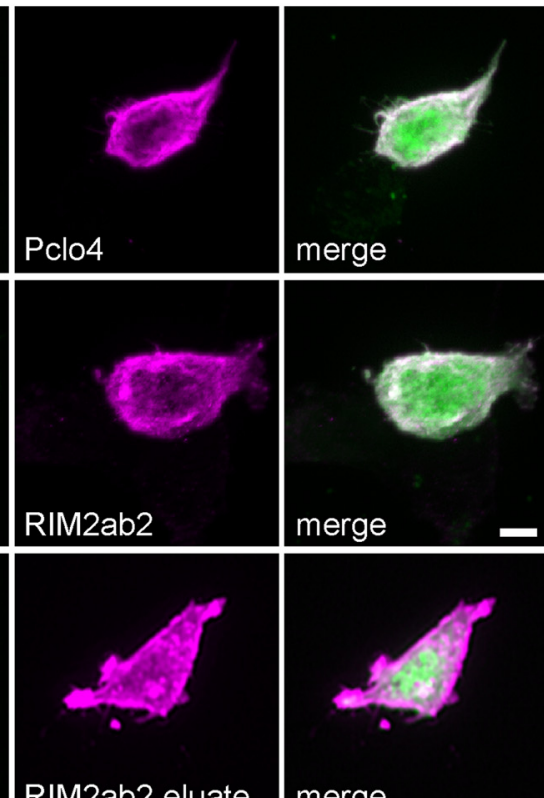

RIM2ab2 eluate
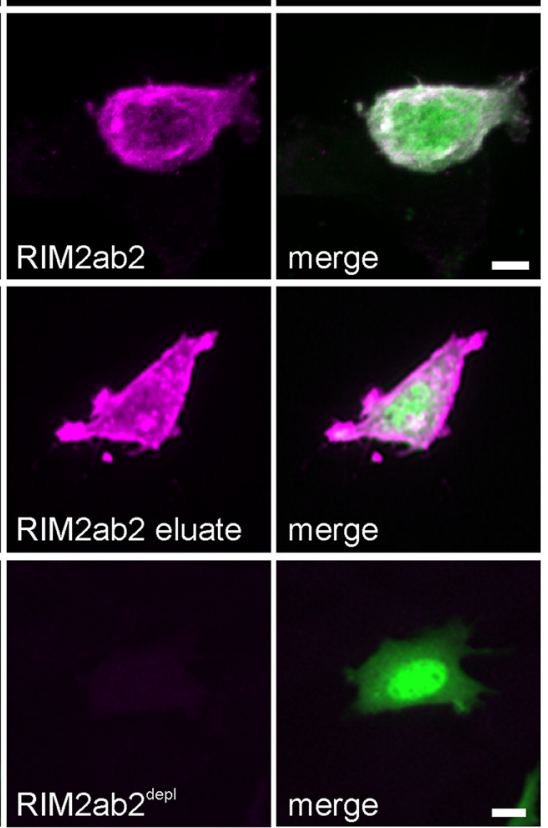

merge

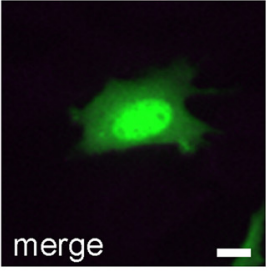

C
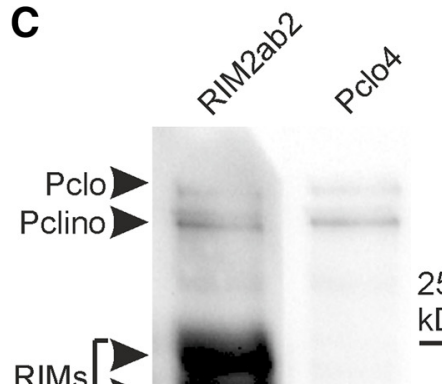

E

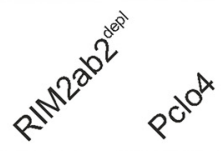

Pclo

Pclino)
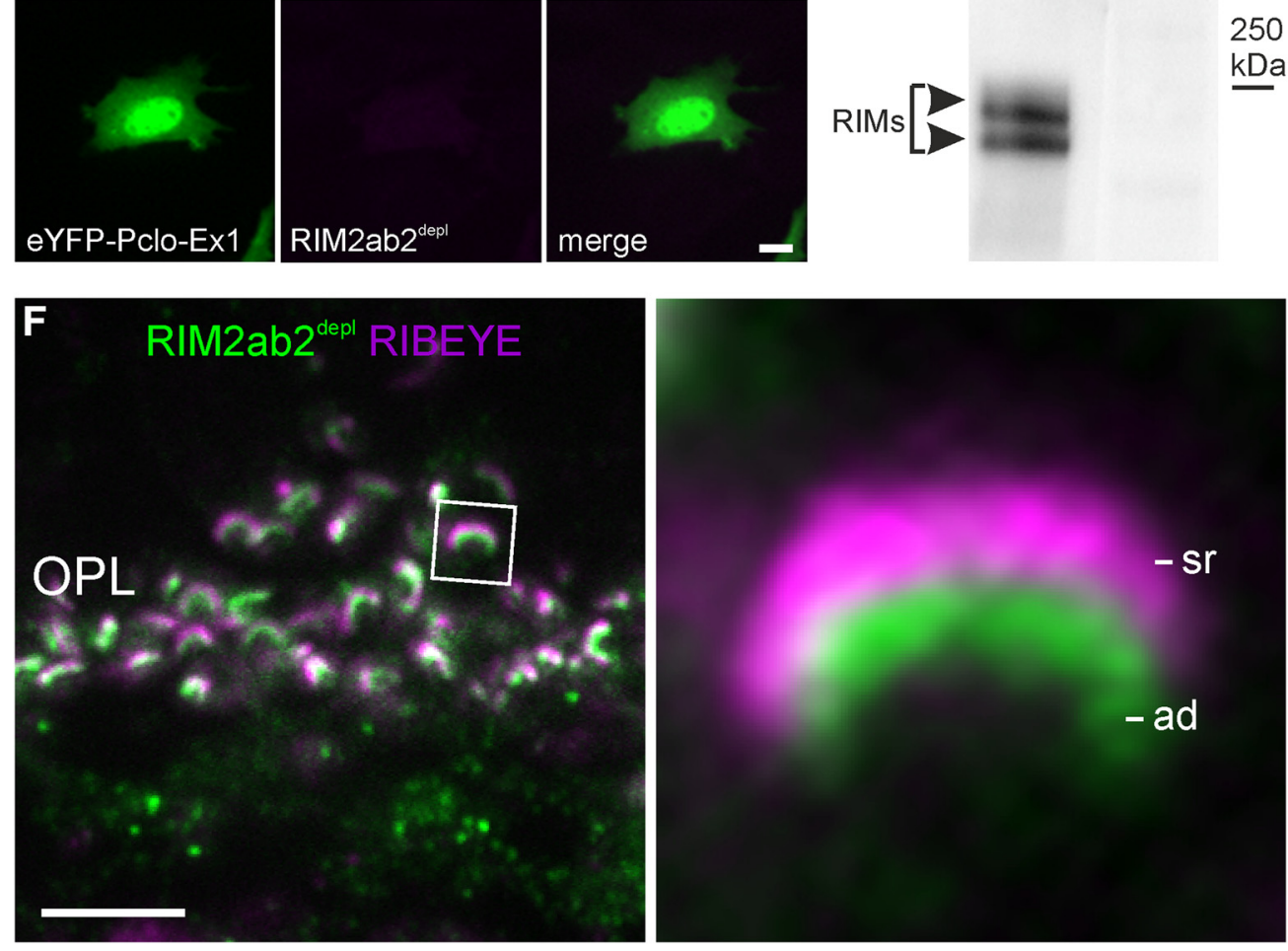

Figure 2. A Piccolo-RIM homology region results in antibody cross-reactivity. A, Amino acid sequence comparison shows a homology region for RIM1 $\alpha$, RIM2 $\alpha$, and Piccolo (Pclo) in the RIM2ab2-binding site. Conserved residues are depicted in black. B. Transfection of HEK293T cells with Piccolo aa1-241 N-terminally fused to eYFP (eYFP-Pclo-aa1-241; green) labeled with an antibody against the Pclo N terminus (Pclo4; magenta; top) and with RIM2ab2 (magenta; bottom). C, Cross-reactivity of RIM2ab2 with Pclo and Piccolino (Pclino) verified in Western blot analysis with mouse retinal synaptosomal fractions. D, Transfection of NIH 3 T3 mouse fibroblasts with eYFP-Pclo-aa1-241 labeled with the eluted RIM2ab2 fraction after binding to the Pclo aa41-aa59 peptide (RIM2ab2 eluate; magenta; top) and with the depleted antibody RIM2ab2 ${ }^{\text {depl }}$ (magenta; bottom). E, Successful abolishment of RIM2ab2 ${ }^{\text {depl }}$ cross-reactivity with Pclo and Pclino verified in Western blot analysis with mouse retinal synaptosomal fractions. F, Immunocytochemical double staining of the OPL of C57BL/6 mice with RIM2ab2 ${ }^{\text {depl }}$ (green) and with an antibody against RIBEYE (magenta). Scale bars in $A, D, E, 5 \mu \mathrm{m}$. 

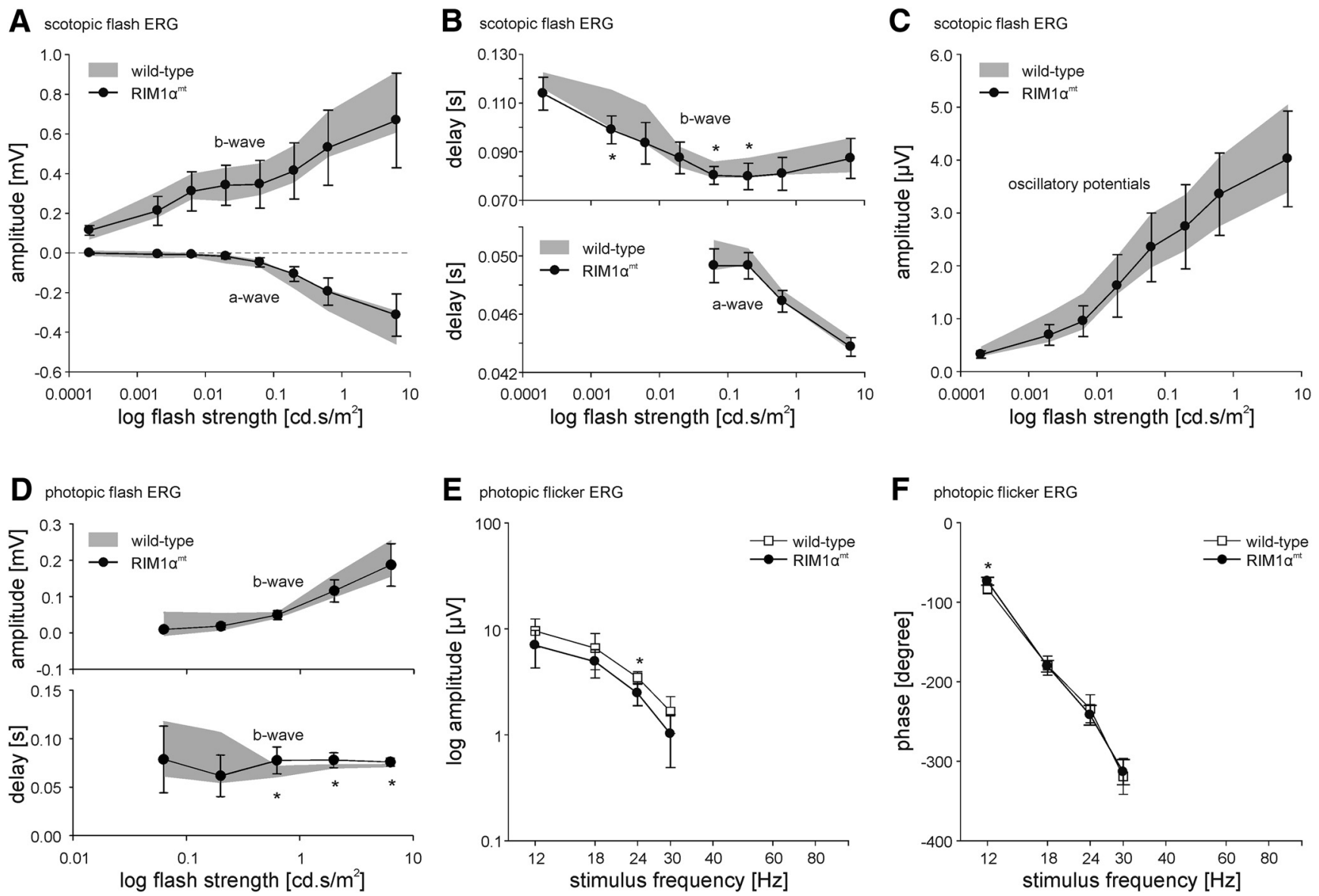

Figure 3. Scotopic and photopic flash and photopic flicker ERGs of wild-type and RIM1 $\alpha^{\mathrm{mt}}$ mice. $A$, Averaged amplitudes of the scotopic a- and b-wave (mean \pm SD) of wild-type (range in gray) and RIM1 $\alpha^{\mathrm{mt}}$ mice (black circles) as a function of stimulus intensity. The a-wave is shown as negative values. $\boldsymbol{B}$, Averaged delays (mean \pm SD) of scotopica-and b-wave of wild-type (range in gray) and RIM1 $\alpha^{\text {mt }}$ mice (black circles) as a function of flash strength. a-wave delay is only shown for flash intensities where the recordings exhibit a noticeable a-wave. C, Averaged amplitude (mean \pm SD) of the OPs of the scotopic flash ERG of wild-type (range in gray) and RIM1 $\alpha^{\mathrm{mt}}$ mice (black circles) as a function of stimulus intensity. $D$, Averaged amplitude and delay (mean \pm SD) of the photopic b-wave of wild-type (range in gray) and RIM1 $\alpha^{\mathrm{mt}}$ mice (black circles) as a function of flash strength. $\boldsymbol{E}$, Averaged amplitudes (mean \pm SD) of the fundamental's amplitude of the photopic flicker ERG of wild-type (white squares) and RIM1 $\alpha^{\mathrm{mt}}$ mice (black circles) as a function of stimulus frequency. $\boldsymbol{F}$, Averaged phases (mean \pm SD) of the fundamental's amplitude of the photopic flicker ERG of wild-type (white squares) and RIM1 $\alpha{ }^{\mathrm{mt}}$ mice (black circles) as a function of stimulus frequency. $\boldsymbol{A}-\boldsymbol{F}$, Statistical differences are indicated by asterisks; ${ }^{*} p<0.05$. For detailed statistics, see Figures 3-1, 3-2, and 3-3 available at https://doi.org/10.1523/JNEUROSCI.2795-16.2017.f3-1, https://doi.org/10.1523/JNEUROSCI.2795-16.2017.f3-2, and https://doi.org/10.1523/JNEUROSCI. 2795-16.2017.f3-3, respectively.

recordings showed similar b-wave amplitudes for both groups and a slightly larger b-wave delay for the RIM1 $1 \alpha^{\mathrm{mt}}$ mice at higher stimulus intensities (Fig. 3D and Fig. 3-2 available at https://doi. org/10.1523/JNEUROSCI.2795-16.2017.f3-2). Photopic a-waves and photopic negative responses were small at all measured intensities and are therefore not shown. In addition to the flash ERGs, we performed photopic flicker ERG measurements and analyzed amplitudes and phases of the first harmonic (fundamental) response components (Fig. 3E,F and Fig. 3-3 available at https://doi.org/10.1523/JNEUROSCI.2795-16.2017.f3-3). Although the amplitude of the first harmonic component was slightly smaller in RIM1 $\alpha^{\mathrm{mt}}$ mice for all recorded stimulus frequencies (Fig. $3 E$ ), the phase was similar in the two genotypes (Fig. $3 F$ ).

To investigate a possible contribution of RIM1 $\alpha$ to exocytosis at the cone photoreceptor ribbon synapse in more detail, we recorded from horizontal cells of RIM $1 \alpha^{\mathrm{mt}}$ mice and measured the tonic release rate at a holding potential of $-60 \mathrm{mV}$ (Fig. $4 A$ ) (Feigenspan and Babai, 2015; Babai et al., 2016). Peak amplitude and interevent interval distributions showed no significant differences (Fig. 4B). Current amplitudes evoked by a full-field stimulation ( $5 \mathrm{~s}$ duration, $130 \mathrm{~W} / \mathrm{cm}^{2}$ ), $20-80 \%$ rise times, and charge transfer values of the off-light response revealed no statistically significant differences between $\operatorname{RIM} 1 \alpha^{\mathrm{wt}}$ and $\operatorname{RIM} 1 \alpha^{\mathrm{mt}}$ mice (Fig. $4 C, D$ ). Finally, current responses evoked by trains of light stimuli ( $500 \mathrm{~ms}, 1 \mathrm{~Hz})$ also showed no significant differences with respect to amplitude and off response charge transfer (Fig. $4 E, F)$. Peak light responses and off responses from wild-type and RIM1 $\alpha^{\mathrm{mt}}$ mice also showed no significant adaptation during the train of 10 stimuli. These findings demonstrate that RIM $1 \alpha$ does not contribute to the regulation of exocytosis at the cone photoreceptor ribbon synapse.

Together with the RT-PCR and immunocytochemical results, the electrophysiological data indicate that RIM1 $\alpha$ levels and their contribution to photoreceptor synaptic transmission in mouse photoreceptors are negligible and that RIM2, presumably $\operatorname{RIM} 2 \alpha$, is the major large RIM variant at mouse photoreceptor ribbon synapses.

\section{Photoreceptors primarily express RIM2 variants, which lack the $\mathrm{N}$-terminal zinc finger domain}

To characterize the photoreceptor-RIM2 protein in more detail, we labeled vertical cryostat sections of the mouse retina with the three RIM2 antibodies: the newly generated RIM2ab2 ${ }^{\text {depl }}$ 
A
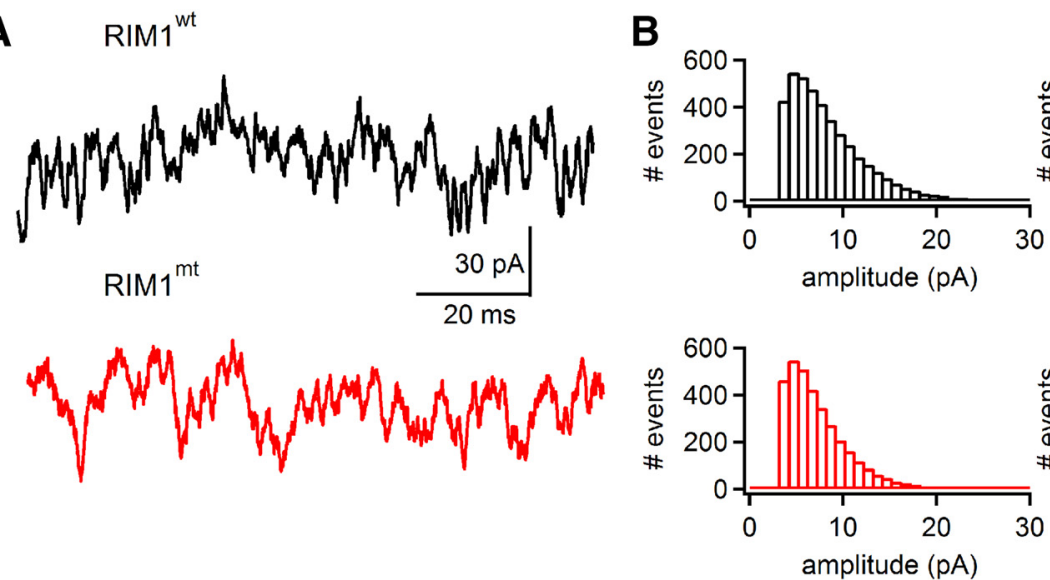

C

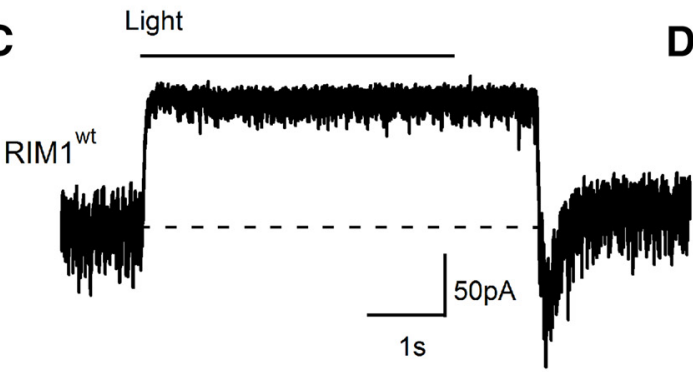

Light

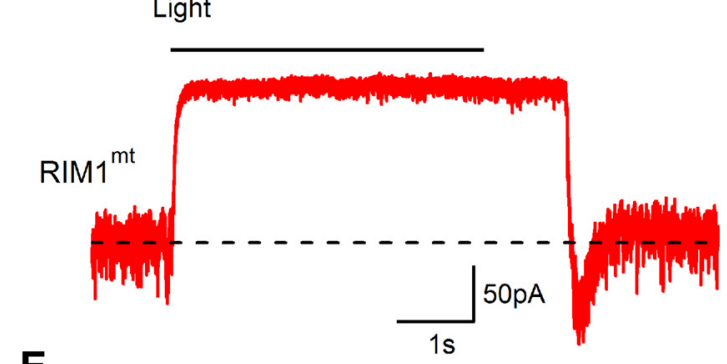

E

D
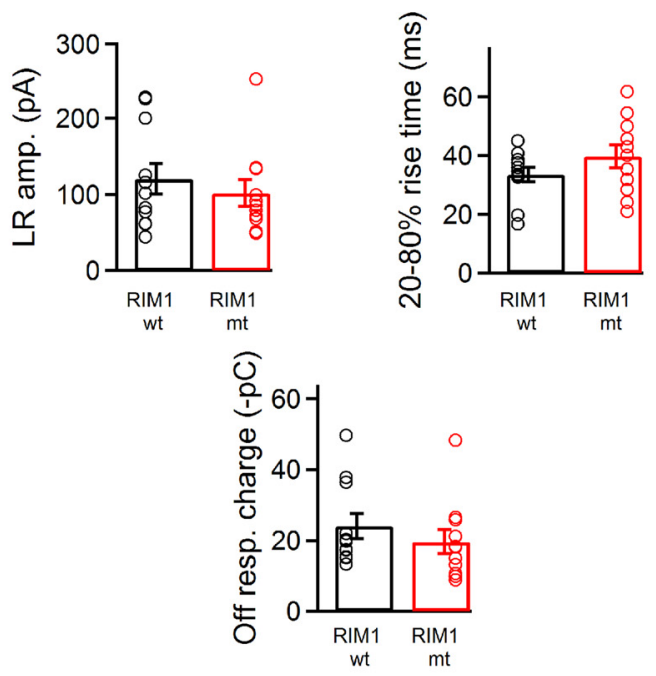
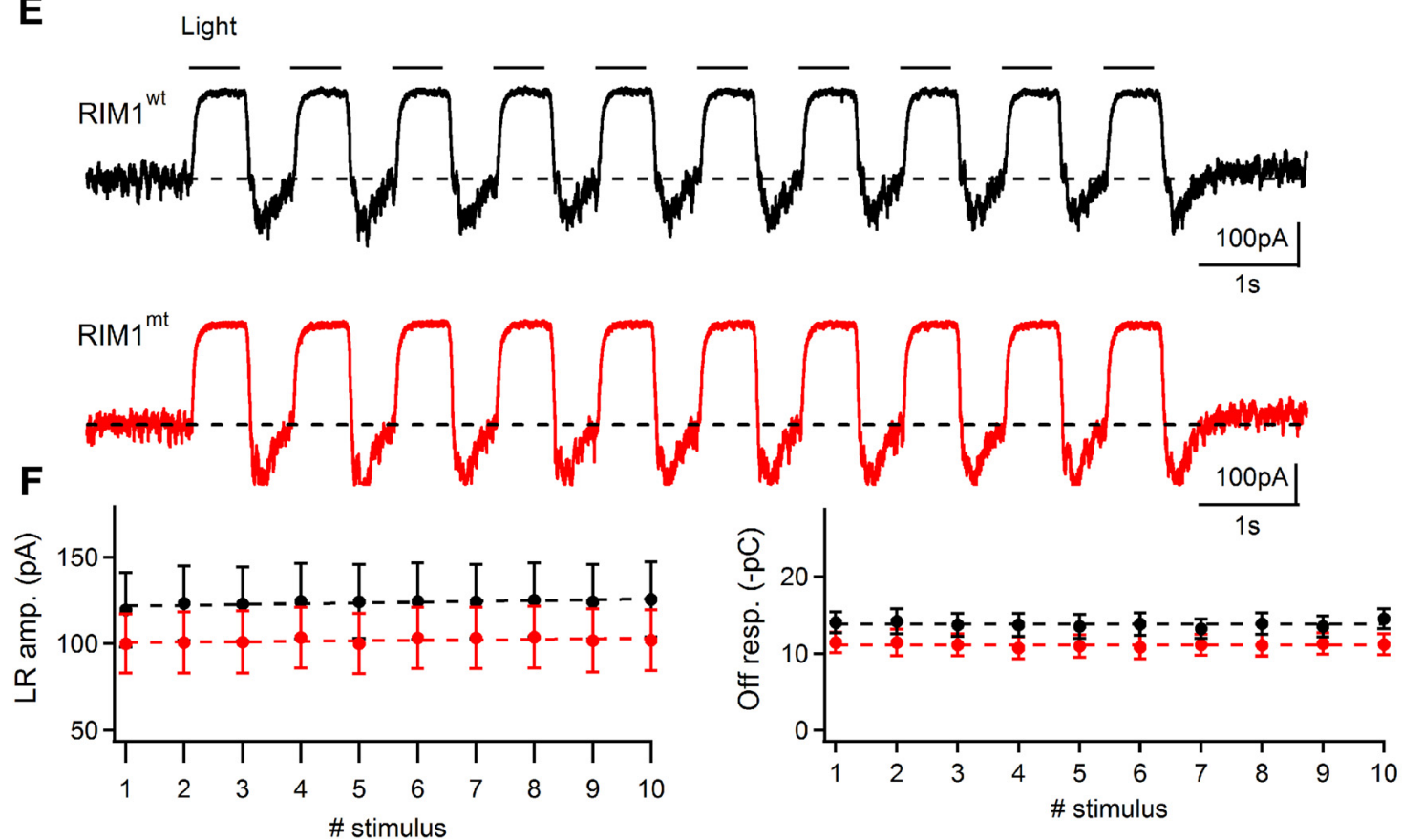

Figure 4. Tonic synaptic activity and light and dark responses in horizontal cells of RIM1 $\alpha^{\text {wt }}$ and RIM1 $\alpha^{\mathrm{mt}}$ mice. $\boldsymbol{A}, \boldsymbol{B}$, Spontaneous events recorded at $-60 \mathrm{mV}$ holding potential from a horizontal cell body in a horizontal slice preparation of a RIM1 $\alpha^{\text {wt }}$ (black trace) and a RIM1 $\alpha^{\text {mt }}$ (red trace) mouse retina. $\boldsymbol{B}$, Amplitude histograms of tonic synaptic events as shown in $\boldsymbol{A}$ and $\boldsymbol{B}$ were constructed from RIM1 $\alpha^{\text {wt }}$ (black trace) and RIM1 $\alpha^{\text {mt }}$ (red trace) retina (bin width: $1 \mathrm{pA}$ ) and interevent interval histograms of EPSCs recorded from RIM1 $\alpha^{\text {wt }}$ (black trace) and RIM1 $\alpha^{\text {mt }}$ (red trace) retina (bin width: $0.5 \mathrm{~ms}$ ). RIM1 $\alpha^{\mathrm{mt}}$ showed no significant difference in the distribution of EPSC amplitudes ( $p=0.67$; Levene's test) and interevent intervals (Figure legend continues.) 

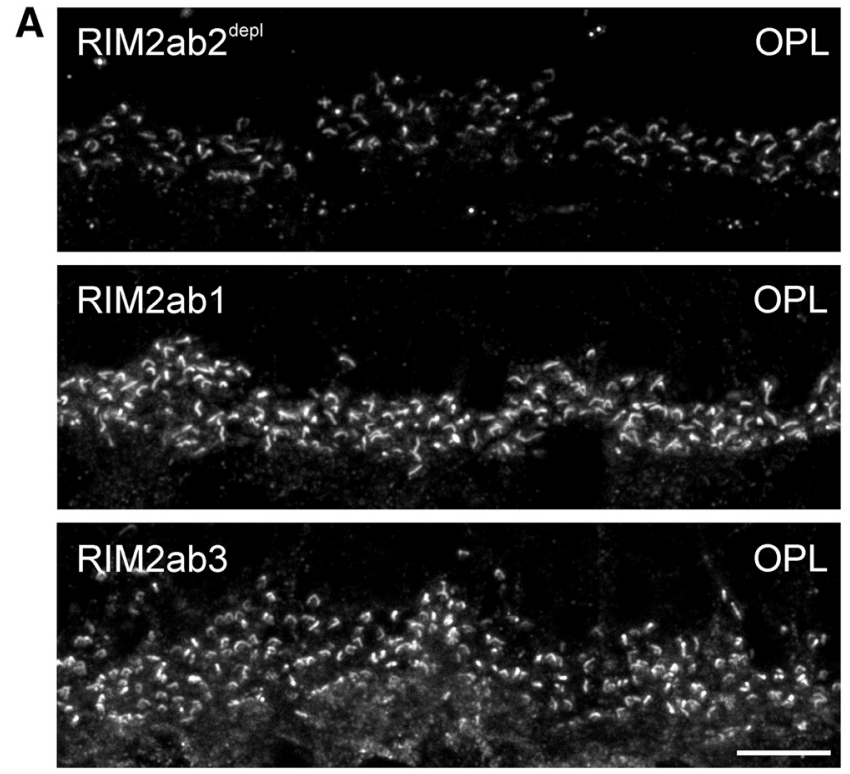

B

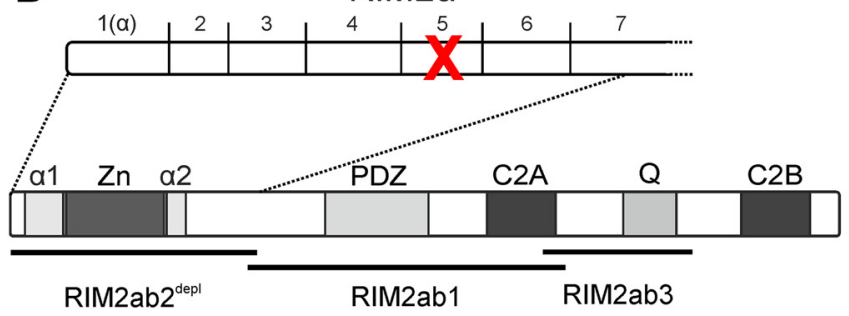

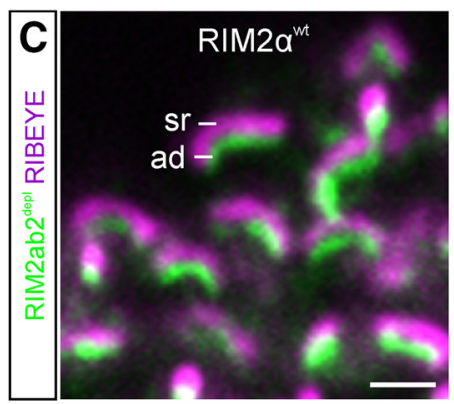
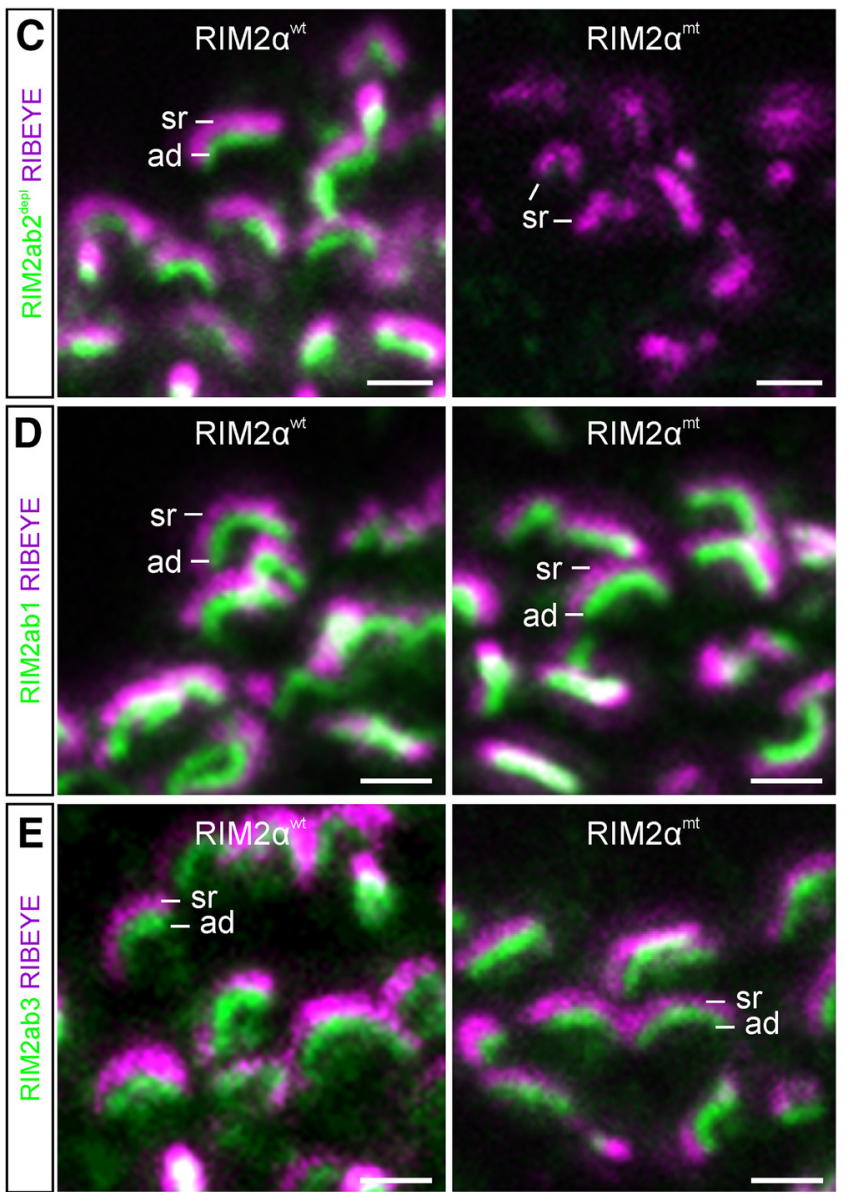

Figure 5. Genetic deletion of RIM2 exon 5 barely affects photoreceptor RIM2. A, Images of vertical sections through the OPL of the C57BL/6 mouse retina stained with the three different RIM2 antibodies (RIM2ab2 ${ }^{\text {depl }}$, RIM2ab1, and RIM2ab3). B, Structure of RIM2 $\alpha$ with binding sites for RIM2ab2 ${ }^{\text {depl }}$, RIM2ab1, and RIM2ab3. Exon structure of the RIM2ab2 ${ }^{\text {depl }}$ epitope region is displayed; exon 5 is genetically deleted in the RIM $2 \alpha^{\mathrm{mt}}$ mouse (red cross). C, Photoreceptor synaptic ribbons in the OPL of the RIM2 $\alpha^{\text {wt }}$ and RIM2 $\alpha^{\mathrm{mt}}$ retina double labeled for RIM2 (RIM2ab2 ${ }^{\text {depl. }}$; green) and RIBEYE (magenta). D, Photoreceptor synaptic ribbons in the OPL of the RIM2 $\alpha^{\text {wt }}$ and RIM $2 \alpha^{\mathrm{mt}}$ retina double labeled for RIM2 (RIM2ab1; green) and RIBEYE (magenta). $\boldsymbol{E}$, Photoreceptor synaptic ribbons in the OPL of the RIM2 $\alpha^{\text {wt }}$ and RIM2 $\alpha{ }^{\mathrm{mt}}$ retina double labeled for RIM2 (RIM2ab3; green) and RIBEYE (magenta). ad, Arciform density; sr, synaptic ribbon. Scale bars: $\boldsymbol{A}, 10 \mu \mathrm{m} ; \boldsymbol{C}-\boldsymbol{E}, 1 \mu \mathrm{m}$.

directed against the N-terminal part of RIM2, RIM2ab1 directed against the central part of RIM2, and RIM2ab3 directed against the $\mathrm{C}$ terminus of RIM2. Staining intensities were compared by taking photographs with the same exposure time (Fig. $5 A$ ). The intensity of the RIM2ab2 ${ }^{\text {depl }}$ staining was much weaker than the RIM2ab1 and the RIM2ab3 staining. To us, this indicated that only a minor fraction of RIM2 in mouse photoreceptor ribbon synapses is full-length RIM $2 \alpha$, whereas the majority of RIM2 might represent alternatively spliced $\operatorname{RIM} 2 \alpha$ and/or RIM2 $\beta$;

$\leftarrow$

(Figure legend continued.) ( $p=0.70$; Mann-Whitney $U$ test). C, Relatively dark-adapted horizontal slice from RIM1 $\alpha^{\text {wt }}$ (black trace) and RIM1 $\alpha^{\text {mt }}$ (red trace) retina stimulated with a $5 \mathrm{~s}$ full-field-light flash. Current changes in response to the light stimulus recorded from horizontal cell bodies at $-60 \mathrm{mV}$ holding potential. $\boldsymbol{D}$, Light response amplitude, rise time, and charge transfer of off-light response values were not significantly different between the two genotypes (light response amplitude: $p=0.49$; rise time: $p=0.21$; charge values of off-light responses $p=0.37$; unpaired $t$ test). $\boldsymbol{E}$, Horizontal slice from RIM1 $\alpha^{\text {wt }}$ (black trace) and RIM1 $\alpha^{\text {mt }}$ (red trace) retina stimulated with a $1 \mathrm{~Hz}$ (500 ms duration) full field light stimulus (10 stimuli). Current changes in response to the train recorded from horizontal cell bodies at $-60 \mathrm{mV}$ holding potential. $F$, Peak light responses and off responses did not show significant adaptation during the course of stimulation as determined by fitting a linear regression line to the data points. Slope values for peak light responses were $0.431 \pm 0.113$ and $0.275 \pm 0.181$ for $\mathrm{RIM} 1 \alpha^{\text {wt }}$ and RIM1 $\alpha^{\mathrm{mt}}$, respectively ( $p=0.4752$; unpaired $t$ test). Slope values for corresponding off responses were $0.009 \pm 0.032$ and $-0.013 \pm 0.027$ ( $p=0.5982$; unpaired $t$ test). $n=11$ cells from 3 animals/genotype. note, however, that we were not able to amplify RIM $2 \beta$ transcript from photoreceptors (Fig. 1A).

To support our hypothesis of a prevalence of alternatively spliced RIM $2 \alpha /$ RIM $2 \beta$ in mouse photoreceptors, we next analyzed a RIM $2 \alpha^{\mathrm{mt}}$ mouse line with a genetic deletion of RIM2 exon 5. This deletion leads to the complete absence of RIM $2 \alpha$ staining in the brain (Schoch et al., 2006). However, because the majority of RIM2 variants in wild-type photoreceptors should lack exon 5 (Fig. $5 B$ ), we assumed that the genetic deletion of RIM2 exon 5 would not have a great impact on overall RIM2 levels in photoreceptors. To test this, we immunocytochemically stained photoreceptor ribbon synapses of RIM $2 \alpha^{\mathrm{wt}}$ and RIM $2 \alpha^{\mathrm{mt}}$ mice with the three RIM2 antibodies (Fig. 5C-E). As expected, the antibody RIM2ab2 ${ }^{\mathrm{depl}}$ produced an arciform density staining in rod photoreceptors, which was weak in the RIM $2 \alpha^{\text {wt }}$ and barely detectable in the RIM $2 \alpha^{\mathrm{mt}}$ (Fig. 5C), whereas both RIM2ab1 (Fig. 5D) and the RIM2ab3 (Fig. 5E) produced a staining of the arciform density comparable in intensity to the RIM $2 \alpha^{\mathrm{wt}}$. Intensity measurements of individual 3D reconstructed horseshoe shaped RIM2ab3 stainings in RIM2 $\alpha^{\mathrm{wt}}$ and RIM $2 \alpha^{\mathrm{mt}}$ rod photoreceptor terminals revealed only a slight, albeit significant, $6 \%$ decrease in overall RIM2 protein levels in the RIM $2 \alpha^{\mathrm{mt}}$ retina (RIM2 $\alpha^{\mathrm{wt}}: 100 \pm 30 \%$ SD; RIM $2 \alpha^{\mathrm{mt}}: 94 \pm 20 \%$ SD; $p<0.001$; Kruskal-Wallis test; $n$ : RIM $2 \alpha^{\text {wt }}=876$ active zones from 3 animals, RIM $2 \alpha^{\mathrm{mt}}=1270$ active zones from 3 
A $R / M 2 \alpha$

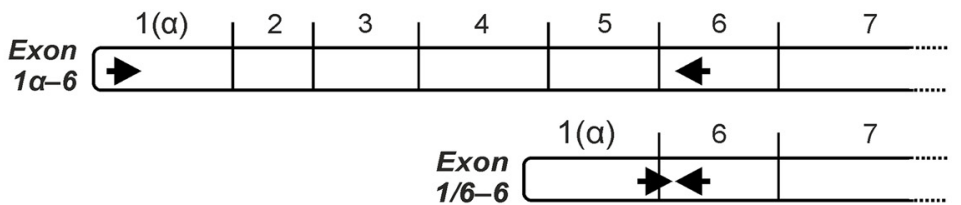

B

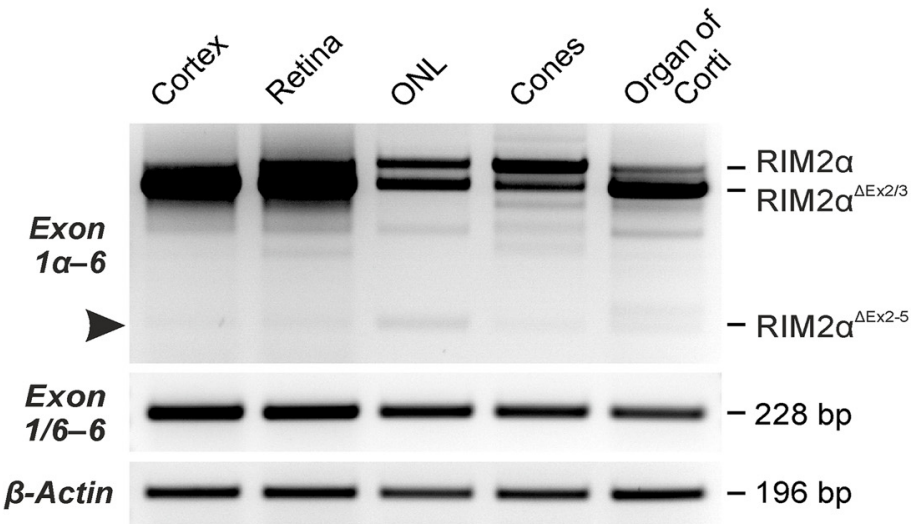

C

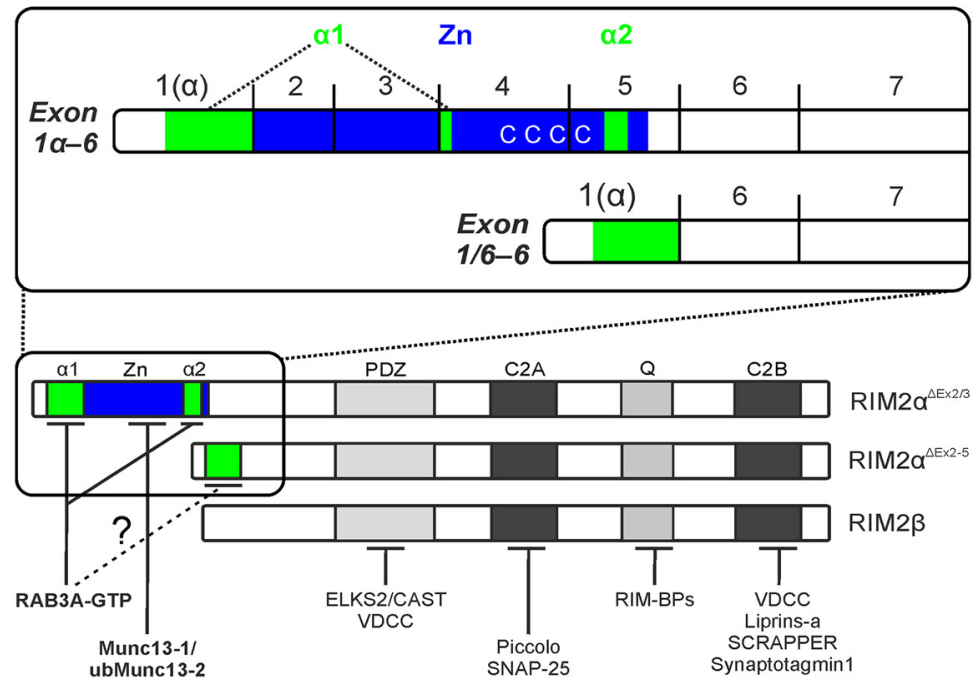

D
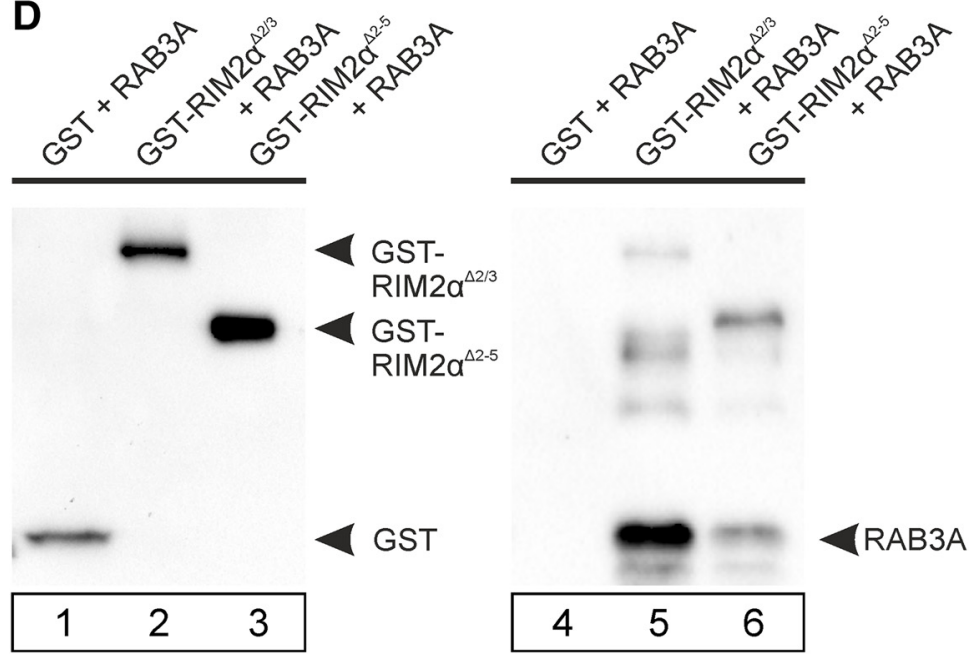

Figure 6. Identification of an alternative RIM2 $\alpha$ transcript lacking exons 2-5 (RIM $2 \alpha^{\Delta \mathrm{Ex} 2-5}$ ). A: Exon structure of the fulllength RIM $2 \alpha$ transcript and an alternative RIM2 $\alpha$ transcript without exons 2-5. Arrows indicate the primer pairs used in $\boldsymbol{B}$. B, RT-PCR of CDNA from RIM2 $\alpha^{\text {wt }}$ cortex, whole retina, ONL, cone photoreceptors (Cones), and organ of Corti. animals). These results support our assumption that only a minor fraction of full-length RIM $2 \alpha$ is abolished in the RIM $2 \alpha^{\mathrm{mt}}$ photoreceptors, whereas the majority of RIM2 remains unaffected.

To test for the expression of alternative RIM $2 \alpha$ transcripts in the retina that lack larger parts of the RIM $2 \alpha \mathrm{N}$ terminusencoding exons, we performed an RTPCR analysis with primers that cover the RIM2ab2 ${ }^{\text {depl }}$ epitope-encoding region (RIM2 exon $1 \alpha-6$; Figs. $5 B, 6 A$ ). This analysis showed the presence of two major RIM $2 \alpha$ splice transcripts in cortex and retina samples, corresponding to a RIM $2 \alpha$ variant containing the full complement of exons $1 \alpha-6$ (RIM $2 \alpha$; Fig. $6 B$ ), and a variant lacking exons 2 and 3 (RIM $2 \alpha^{\Delta \mathrm{Ex} 2 / 3}$; Fig. $6 B)$, representing the major splice variant in the brain (Fukuda, 2003; Wang and Südhof, 2003). In addition, we detected a weak band of $271 \mathrm{bp}$ in both samples, representing an in-frame RIM $2 \alpha$ transcript with exon $1 \alpha$ directly spliced to exon 6 (RIM $2 \alpha^{\Delta \mathrm{Ex} 2-5}$, Fig. $6 B$, arrowhead). The resulting translational product would lead to a RIM $2 \alpha$ variant lacking a major part of the $\mathrm{N}$ terminus (Fig. $6 C$ ). RT-PCR with exon $1 / 6$ overlapping primers (RIM2 exon 1/6-6; Fig. 6B) verified the existence of this alternative transcript in all samples (RIM $2 \alpha^{\Delta \text { Ex2-5} ; ~ F i g . ~} 6 B$ ). At this point, we cannot say whether the RIM $2 \alpha^{\Delta \text { Ex2-5 }}$ transcript is the only transcript leading to the large fraction of $\mathrm{N}$-terminally truncated RIM $2 \alpha$ variants in mouse photoreceptors. However, RTPCR analysis using different combinations of multiple forward and reverse primers covering the first 10 RIM2 exons (data not shown) did not reveal the pres-

$\leftarrow$

Primers spanning RIM2 exons $1 \alpha-6$ amplified the full-length RIM $2 \alpha$ transcript, a splice variant lacking exons 2 and 3 $\left(\right.$ RIM $2 \alpha^{\Delta \mathrm{Ex} 2 / 3}$ ) and a shorter amplicon (arrowhead) corresponding to a splice variant without exons $2-5\left(\right.$ RIM $\left.2 \alpha^{\Delta \text { Ex2-5 }}\right)$. RT-PCR with a forward primer designed to bridge the exon border $1 / 6$ verified the presence of the RIM $2 \alpha^{\Delta \mathrm{Ex} 2-5}$ transcript. $\beta$-Actin primers were used as a loading control. $C$, Schematic representation of the prevalent $\operatorname{RIM} 2 \alpha$ protein variant in the brain (transcript RIM $2 \alpha^{\Delta \mathrm{Ex} 2 / 3}$ ), the newly identified RIM $2 \alpha$ variant (transcript RIM $2 \alpha^{\Delta \mathrm{E} \times 2-5}$ ), and RIM $2 \beta$ with their interaction domains and known binding partners. The inset depicts the exon structure of both RIM $2 \alpha$ transcripts; coding regions for the two $\alpha$-helices of the RAB3A-binding domain ( $\alpha 1$ and $\alpha 2$; green) and for the zinc finger domain ( $\mathrm{Zn}$; blue) with its conserved cysteine residues are shown. $\boldsymbol{D}$, Pulldowns of GSTtagged N-terminal regions of RIM2 $\alpha$ (GST-RIM $2 \alpha^{\Delta \text { Ex2/3 }}$ and GST-RIM $2 \alpha^{\Delta \mathrm{Ex} 2-5}$ ) with RAB3A in the presence of GTP. Samples were analyzed by immunoblotting with antibodies against GST and RAB3A. $\alpha 1$ and $\alpha 2$, RAB3A-binding domains; $\mathrm{mt}$, mutant; Zn, zinc finger. 

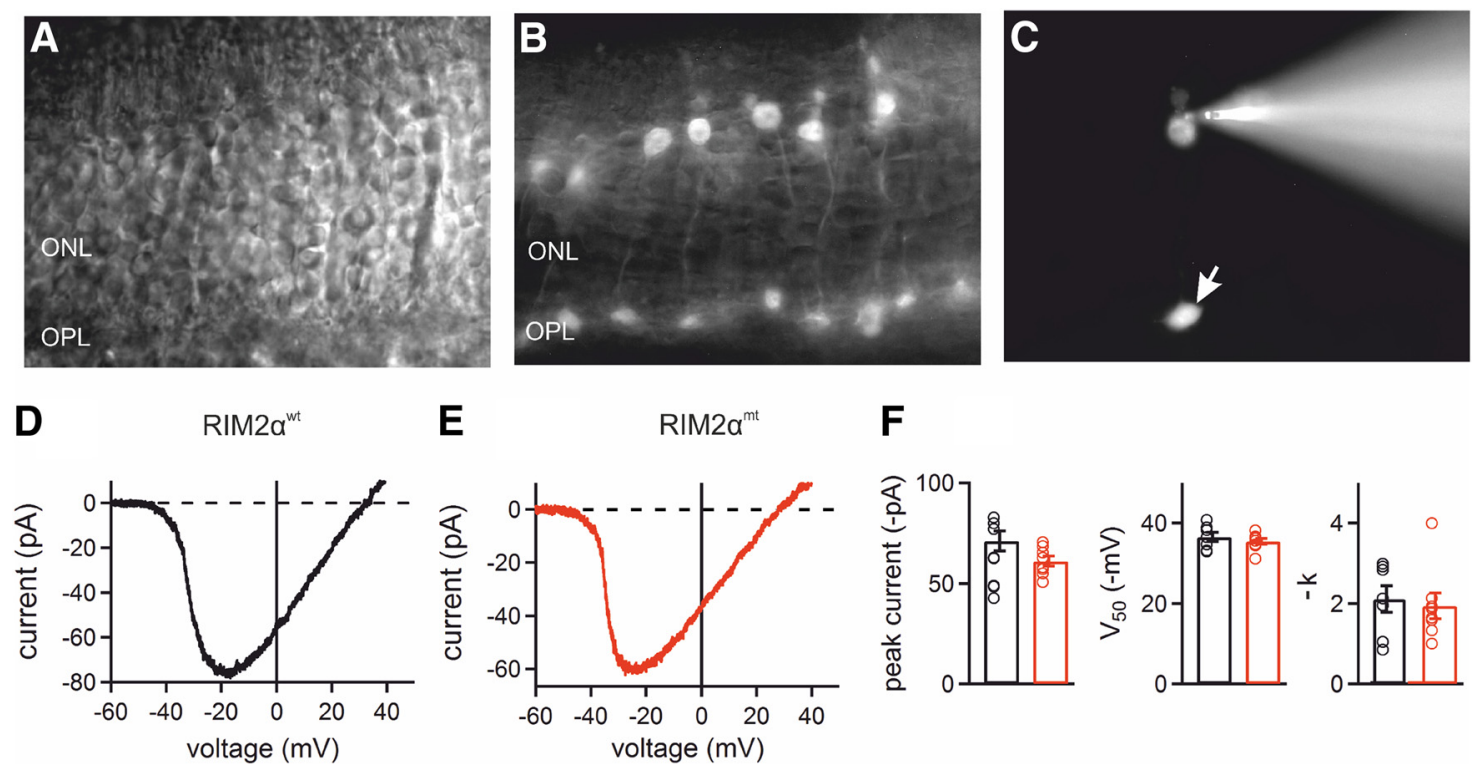

Figure 7. Whole-cell $\mathrm{Ca}^{2+}$ current measurements from RIM $2 \alpha^{\text {wt }}$ and RIM $2 \alpha^{\text {mt }}$ mice. $A, 0$ NL in a vertical slice from a RIM2 $\alpha^{\text {wt }} /$ Rac3-eGFP retina visualized with Dodt contrast optics. $\boldsymbol{B}$, Corresponding fluorescence image revealing eGFP-labeled cone photoreceptors. $\boldsymbol{C}$, Individual cone photoreceptor injected with $100 \mu \mathrm{m}$ Alexa Fluor 568 during the patch-clamp recording. The electrode is attached to the cell body of the cone photoreceptor; the arrow indicates its synaptic terminal in the OPL. D, E, Voltage-dependent $C a^{2+}$ currents induced by a voltage ramp protocol ( -60 to $+50 \mathrm{mV}, 0.11 \mathrm{mV} \mathrm{ms}^{-1}$ ) recorded from cone photoreceptor cell bodies in the RIM2 $\alpha$ wt $/$ Rac3-eGFP retina and in the RIM $2 \alpha$ mt $/$ Rac3-eGFP retina. F, Parameters obtained by fitting a Boltzmann distribution to the voltage-dependent activation of $\mathrm{Ca}^{2+}$ channels in RIM $2 \alpha^{\text {wt }}$ and RIM $2 \alpha^{\mathrm{mt}}$ cone photoreceptors. Values were not significantly different (peak current: $p=0.064 ; V_{50}: p=0.39$; $k: p=0.92$; unpaired $t$ test). $n=9$ cells from 3 RIM $2 \alpha^{\text {wt }}$ animals and 14 cells from 4 RIM $2 \alpha^{\text {mt }}$ animals. Scale bars in $\boldsymbol{B}$ and $\boldsymbol{F}, 20 \mu \mathrm{m}$.

ence of any other alternative RIM $2 \alpha$ transcript than the one we show here.

Both the antibody experiments (Fig. 5) and the identification of an alternative RIM $2 \alpha^{\Delta \mathrm{Ex} 2-5}$ transcript (Fig. $6 A, B$ ) indicate that the majority of RIM2 in photoreceptors lacks the Zn domain (blue) and part of the RAB3A-binding domain (green; composed of the $\alpha 1$ and $\alpha 2$ domain), which are responsible for binding of Munc13-1/ubMunc13-2 and RAB3A, respectively (Fig. 6C) (Wang et al., 1997; Betz et al., 2001; Fukuda, 2003; AndrewsZwilling et al., 2006). The alternatively spliced RIM $2 \alpha$ would therefore be structurally comparable to RIM $2 \beta$ (Fig. 6C), which lacks the ability to interact with the photoreceptor-specific ubMunc13-2 and RAB3A (Cooper et al., 2012). However, in contrast to the complete lack of the RAB3A-binding domain in $\operatorname{RIM} 2 \beta$, part of this binding domain is still present in any alternatively spliced RIM $2 \alpha$ because the $\alpha 1$-domain of the RAB3Abinding domain is encoded in RIM $2 \alpha$ exon 1 (Fig. 6C). Therefore, we investigated RAB3A binding to RIM $2 \alpha^{\Delta \mathrm{Ex} 2-5}$ with a GST pulldown assay (Fig. 6D). GST fusion proteins containing the RIM $2 \alpha$ $\mathrm{N}$ terminus but lacking either exons 2 and 3 (GST-RIM $2 \alpha^{\Delta 2 / 3}$ ) or exons $2-5$ (RIM $2 \alpha^{\Delta \text { Ex2-5}}$ ) were used as immobilized bait proteins; GST alone served as negative control. Overexpressed RAB3A was used as soluble prey protein. Because the binding of RAB3A to RIM occurs only in its activated form, the pulldown experiments were performed in the presence of GTP $\gamma \mathrm{S}$. Both GST-RIM $2 \alpha^{\Delta 2 / 3}$ and GST-RIM $2 \alpha^{\Delta \mathrm{Ex} 2-5}$, but not GST alone, were able to pull down RAB3A-GTP $\gamma S$ (Fig. 6D). In two of four independent experiments, binding of RAB3A-GTP $\gamma \mathrm{S}$ to GST-RIM $2 \alpha^{\Delta \mathrm{Ex} 2-5}$, however, seemed less efficient than to GST-RIM $2 \alpha^{\Delta 2 / 3}$, as judged by a weaker RAB3A staining (Fig. $6 D$, lanes 5,6$)$. We also confirmed the lack of ubMunc13-2 binding to RIM $2 \alpha^{\Delta \mathrm{Ex} 2-5}$ in the absence of the zinc finger (data not shown).

From these findings, we conclude that the prevalent RIM2 in mouse photoreceptors is unable to bind ubMunc13-2 and that
Table 1. Biophysical membrane properties of cone photoreceptors from RIM2 $\alpha^{\text {wt }}$ and $\mathrm{RIM} 2 \alpha^{\mathrm{mt}}$ mouse retinas

\begin{tabular}{lccc}
\hline & $\begin{array}{l}\text { RIM2 } \alpha^{\mathrm{wt}} \\
(n=7)\end{array}$ & \multicolumn{1}{l}{$\begin{array}{l}\mathrm{RIM2} \alpha^{\mathrm{mt}} \\
(n=8)\end{array}$} & \multicolumn{1}{c}{$p$} \\
\hline Membrane capacitance $(\mathrm{pF})$ & $4.23 \pm 0.51$ & $4.16 \pm 0.49$ & 0.93 \\
Input resistance $(\mathrm{G} \Omega)$ & $3.05 \pm 0.70$ & $2.42 \pm 0.53$ & 0.47 \\
Access resistance $(\mathrm{M} \Omega)$ & $44.07 \pm 4.90$ & $37.81 \pm 4.83$ & 0.38 \\
\hline
\end{tabular}

$p$-values were obtained with unpaired $t$ test.

binding of RAB3A-GTP $\gamma$ S is probably either weak (RIM $2 \alpha$ splice variant) or not possible (RIM2 $\beta)$.

\section{Photoreceptor synaptic transmission does not depend on full-length RIM $2 \alpha$}

Next, we used electrophysiology to examine RIM $2 \alpha^{\mathrm{mt}}$ photoreceptors, which only lack full-length RIM $2 \alpha$. A recent study on the function of RIMs at photoreceptor ribbon synapses examined a conditional double knock-out of RIM1 and RIM2 from rod photoreceptors (Grabner et al., 2015). The results of that study showed that RIM1/2 facilitate $\mathrm{Ca}^{2+}$ entry into rod photoreceptor terminals and, in turn, $\mathrm{Ca}^{2+}$-evoked release by modulating $\mathrm{Ca}^{2+}$ channel openings (Grabner et al., 2015).

To test whether the slight reduction in overall RIM2 levels in the OPL has any influence on $\mathrm{Ca}^{2+}$ channel function in photoreceptors, we recorded $\mathrm{Ca}^{2+}$ currents from fluorescently labeled cone photoreceptors of RIM $2 \alpha^{\text {wt }} /$ Rac3-eGFP and RIM $2 \alpha^{\mathrm{mt}} /$ Rac3-eGFP mice in a vertical slice preparation (Fig. $7 A, B$ ). During recording, cone photoreceptors were filled with Alexa Fluor $568(100 \mu \mathrm{M})$ to confirm their identity (Fig. 7C). Voltagedependent $\mathrm{Ca}^{2+}$ currents were measured with a voltage ramp protocol running from -60 to $+50 \mathrm{mV}\left(0.11 \mathrm{mV} \mathrm{ms}^{-1}\right)$. The corresponding current-voltage relations are plotted for RIM $2 \alpha^{\mathrm{wt}}$ and RIM $2 \alpha^{\mathrm{mt}}$ mice in Figure 7, D and $E$, respectively. Currents were fitted with a Boltzmann distribution (see Materials and 

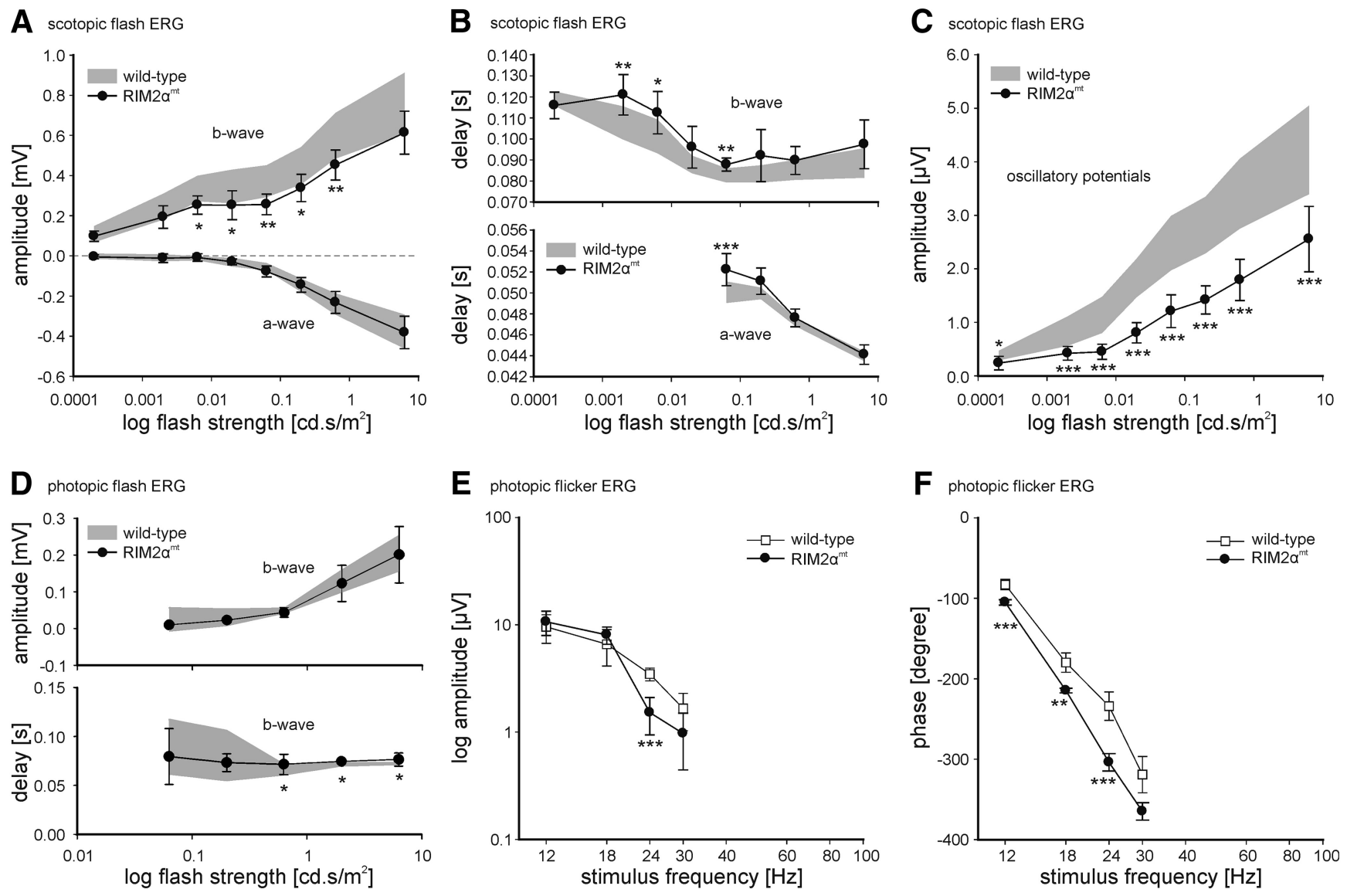

Figure 8. Scotopic and photopic flash and photopic flicker ERGs of wild-type and RIM $2 \alpha^{\mathrm{mt}}$ mice. $A$, Averaged amplitudes (mean \pm SD) of the scotopic a- and b-wave of wild-type (range in gray) and RIM $2 \alpha^{\mathrm{mt}}$ mice (black circles) as a function of flash strength. The a-wave amplitude is given as negative values. $\boldsymbol{B}$, Averaged delays (mean \pm SD) of the scotopic a- (bottom) and b-wave (top) of wild-type (range in gray) and RIM $2 \alpha^{\mathrm{mt}}$ mice (black circles) as a function of flash strength. a-wave delays are only shown at the four highest flash strengths where a prominent a-wave is present. C, Averaged amplitudes (mean \pm SD) of the OPs of the scotopic flash ERG of wild-type (range in gray) and RIM $2 \alpha^{\mathrm{mt}}$ mice (black circles) as a function of flash strength. $\boldsymbol{D}$, Averaged amplitudes (mean \pm SD) and delays (mean \pm SD) of the a- and b-wave of the photopic flash ERG at a flash strength of $6.3 \mathrm{~cd} . \mathrm{s} / \mathrm{m}^{2}$ of wild-type (black columns) and RIM $2 \alpha^{\mathrm{mt}}$ mice (gray columns).The $y$-axis either shows the delay in seconds or the amplitude in millivolts. $\boldsymbol{E}$, Averaged amplitudes (mean \pm SD) of the photopic flicker ERG of wild-type (white squares) RIM2 $\alpha^{\mathrm{mt}}$ mice (black circles) as a function of stimulus frequency. $\boldsymbol{F}$, Averaged phases (mean \pm SD) of the photopic flicker ERG of wild-type (white squares) RIM $2 \alpha^{\mathrm{mt}}$ mice (black circles) as a function of stimulus frequency. In $\boldsymbol{A}-\boldsymbol{F}$, statistical differences are indicated by asterisks: ${ }^{*} p<0.05,{ }^{* *} p<0.01,{ }^{* * *} p<0.001$. For detailed statistics, see Figures $8-1,8-2$, and $8-3$ available at https://doi.org/10.1523/JNEUROSCI. 2795-16.2017.f8-1, https://doi.org/10.1523/JNEUROSCI.2795-16.2017.f8-2, and https://doi.org/10.1523/JNEUROSCI.2795-16.2017.f8-3, respectively.

Methods) and parameters of the fit were not significantly different between the two genotypes (Fig. $7 F$ ). The peak current amplitude showed a tendency to be reduced in RIM $2 \alpha^{\mathrm{mt}}$ mice, albeit without statistical significance. Furthermore, fundamental biophysical parameters (i.e., membrane capacitance, input resistance, and access resistance) did not differ between RIM $2 \alpha^{\mathrm{wt}}$ and RIM $2 \alpha^{\mathrm{mt}}$ cone photoreceptors (Table 1).

Next, we performed ERG recordings as a readout of retinal function and photoreceptor synaptic transmission. Figure 8, $A-C$, shows a comparison of the mean amplitudes and delays of the scotopic a-wave, b-wave, and OPs of the wild-type and RIM $2 \alpha^{\mathrm{mt}}$ mice. In both groups, the a-wave amplitudes were similar over all measured light intensities and the b-wave amplitudes were slightly reduced in the RIM $2 \alpha^{\mathrm{mt}}$ mice (Fig. $8 A$ and Fig. 8-1 available at https://doi.org/10.1523/JNEUROSCI.2795-16.2017. f8-1). The delay of the scotopic a- and b-wave was slightly increased in the RIM $2 \alpha^{\mathrm{mt}}$ compared with the wild-type mice at lower flash strengths (Figs. 8B and Fig. 8-1 available at https://doi.org/10.1523/ JNEUROSCI.2795-16.2017.f8-1). The amplitudes of the OPs were consistently smaller in the RIM $2 \alpha^{\mathrm{mt}}$ than in the wild-type mice (Figs. 8C and Fig. 8-1 available at https://doi.org/10.1523/ JNEUROSCI.2795-16.2017.f8-1). Figure 8D summarizes the am- plitudes and delays of the cone-driven photopic flash ERG (flash strength $6.3 \mathrm{~cd} . \mathrm{s} / \mathrm{m}^{2}$ ). Although the amplitude of the photopic b-wave did not differ between the wild-type and RIM $2 \alpha^{\mathrm{mt}}$ mice, the b-wave delay was significantly larger for RIM $2 \alpha^{\mathrm{mt}}$ mice at higher flash intensities (Fig. 8-2 available at https://doi.org/10. 1523/JNEUROSCI.2795-16.2017.f8-2). We also measured the photopic flicker ERG, which is characterized by its amplitude and phase as a function of stimulus frequency (Fig. $8 E, F$ ). The amplitudes were similar in the two groups (Fig. $8 E$ and Fig. 8-3); the phase was consistently larger in the RIM $2 \alpha^{\mathrm{mt}}$ than in the wildtype mice (Figs. 8F, 8-3 available at https://doi.org/10.1523/ JNEUROSCI.2795-16.2017.f8-3). Together, the results from the ERG recordings suggest that RIM $2 \alpha$ deficiency results in a moderate change of the physiology of the photoreceptor ribbon synapse. Assuming that OPs mainly originate from amacrine cell activity (Frishman, 2006), the data suggest that the physiology of these cells is affected in the RIM $2 \alpha^{\mathrm{mt}}$ mice.

In a final set of experiments, we investigated synaptic release from cone photoreceptor synapses in RIM $2 \alpha^{\mathrm{wt}}$ and RIM $2 \alpha^{\mathrm{mt}}$ mice by recording from postsynaptic horizontal cells in a horizontal slice preparation of the retina at a holding potential of -60 $\mathrm{mV}$. Under relatively dark-adapted conditions, horizontal cells in 
A

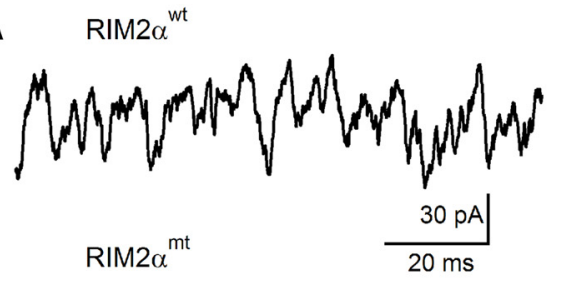

B
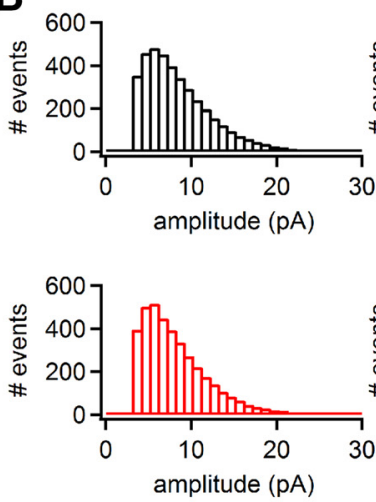
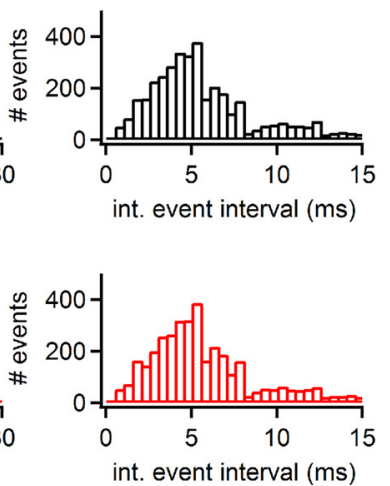

int. event interval (ms)
C
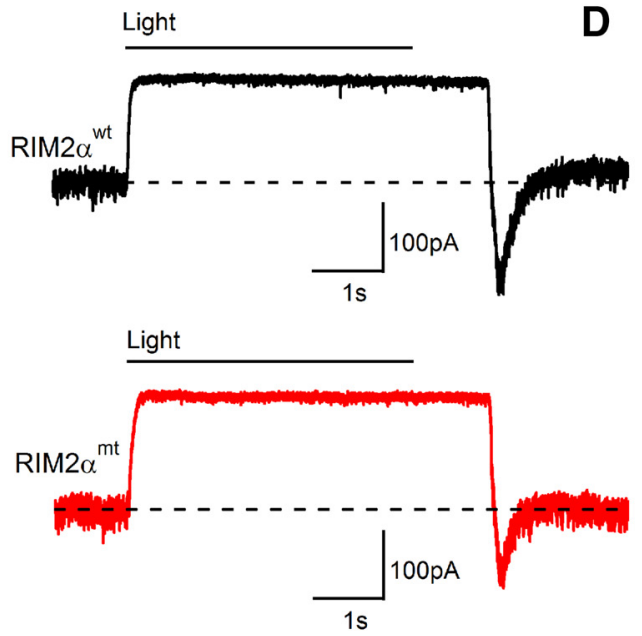

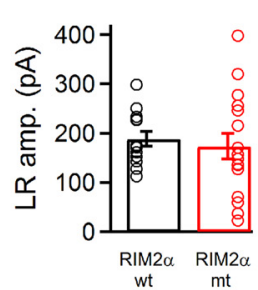

wt $\mathrm{mt}$

$\mathbf{E}$
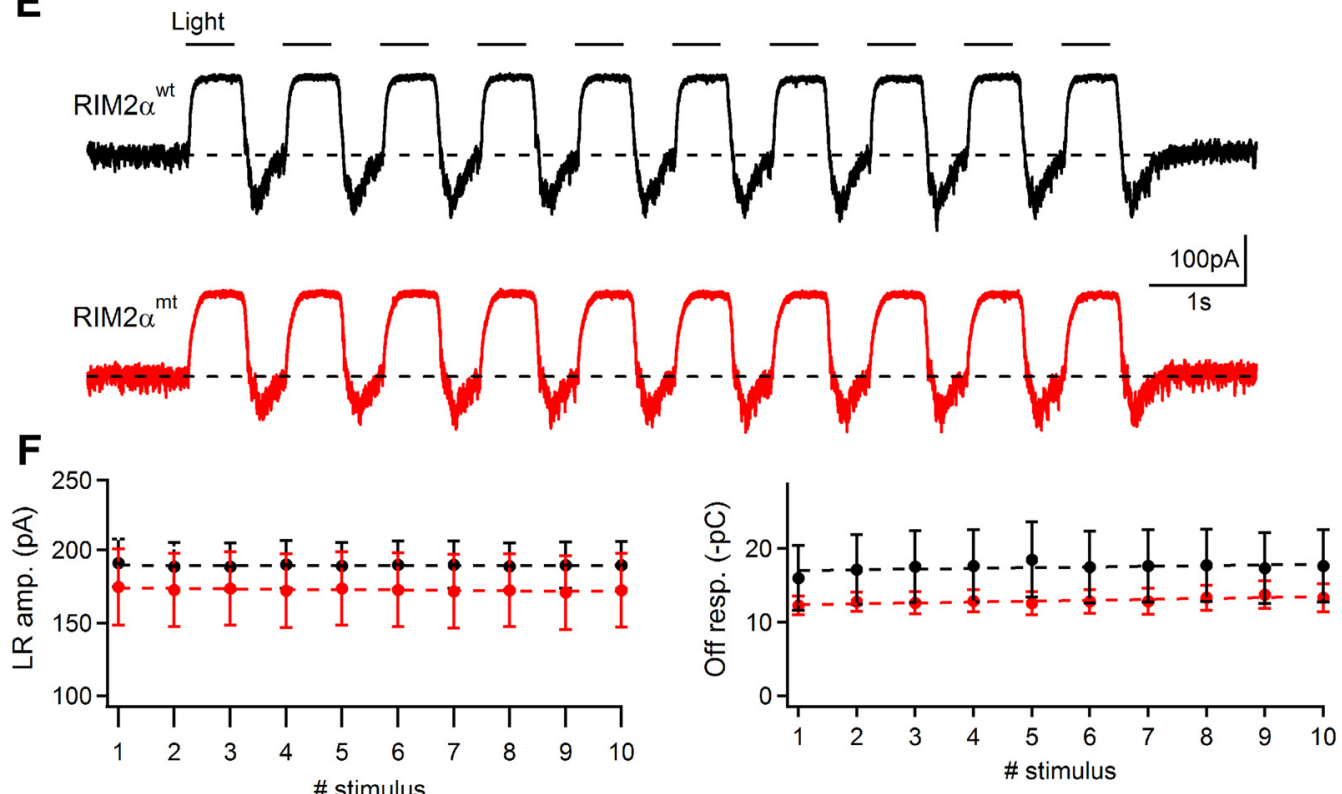

Figure 9. Tonic synaptic activity and light and dark responses in horizontal cells of RIM2 $\alpha^{\mathrm{wt}}$ and RIM2 $\alpha^{\mathrm{mt}}$ mice. $A, B$, Tonic synaptic events recorded at $-60 \mathrm{mV}$ holding potential from a horizontal cell body in a horizontal slice preparation of a RIM $2 \alpha^{\text {wt }}$ (black trace) and a RIM $2 \alpha^{\mathrm{mt}}$ (red trace) mouse retina. $\boldsymbol{B}$, Amplitude histograms of tonic synaptic events as shown in $\boldsymbol{A}$ and $\boldsymbol{B}$ were constructed from RIM $2 \alpha^{\text {wt }}$ (black trace) and RIM $2 \alpha^{\text {mt }}$ (red trace) retina (bin width: $1 \mathrm{pA}$ ) and interevent interval histograms of EPSCs recorded from RIM $2 \alpha^{\text {wt }}$ (black trace) and RIM2 $\alpha^{\mathrm{mt}}$ (red trace) retina (bin width: $0.5 \mathrm{~ms}$ ). RIM $2 \alpha^{\mathrm{mt}}$ showed no significant difference in the distribution of EPSC amplitudes ( $p=0.87$; Levene's test) and interevent intervals ( $p=0.80$; Mann-Whitney $U$ test). C, Relatively dark-adapted horizontal slice from RIM $2 \alpha^{\text {wt }}$ (black trace) and RIM $2 \alpha^{\mathrm{mt}}$ (red trace) retina stimulated with a $5 \mathrm{~s}$ full-field light flash. Current changes in response to the light stimulus recorded from horizontal cell bodies at $-60 \mathrm{mV}$ holding potential. $\boldsymbol{D}$, Light response amplitude and $20-80 \%$ rise time were not significantly different between the two genotypes (light response amplitude: $p=0.65$; rise time: $p=0.26$; unpaired $t$ test), but the charge transfer during the off responses was significantly smaller in $\operatorname{RIM} 2 \alpha^{\mathrm{mt}}$ ( $p=0.037$; unpaired $t$ test). $n=13$ cells from 3 RIM2 $\alpha^{\text {wt }}$ animals and 16 cells from 3 RIM2 $\alpha^{\text {mt }}$ animals. E, Relatively dark-adapted horizontal slice from RIM2 $\alpha^{\text {wt }}$ (black trace) and RIM2 $\alpha^{\text {mt }}$ (red trace) retina stimulated with a $1 \mathrm{~Hz}$ ( $500 \mathrm{~ms}$ duration) full-field light stimulus (10 stimuli). Current changes in response to the train recorded from horizontal cell bodies at $-60 \mathrm{mV}$ holding potential. (Figure legend continues.) 
both RIM $2 \alpha^{\text {wt }}$ and RIM $2 \alpha^{\mathrm{mt}}$ mice showed tonic synaptic activity (Fig. 9A), which was caused by the continuous release of glutamate from cone photoreceptors in the dark. Amplitudes of synaptic events, as determined from the respective amplitude distributions, were not significantly different between RIM $2 \alpha^{\text {wt }}$ and RIM $2 \alpha^{\mathrm{mt}}$ mice (Fig. 9B). The frequency of tonic EPSCs, as determined from interevent interval histogram, also showed no significant difference between RIM $2 \alpha^{\mathrm{wt}}$ and RIM $2 \alpha^{\mathrm{mt}}$ mice (Fig. 9B).

To determine whether the horizontal cell light response was affected in RIM $2 \alpha^{\mathrm{mt}}$ mice, we used a full-field light flash $(5 \mathrm{~s}$ duration, $130 \mathrm{~W} / \mathrm{cm}^{2}$ ). As a response to the onset of light, release of glutamate was reduced in both RIM $2 \alpha^{\mathrm{wt}}$ and RIM $2 \alpha^{\mathrm{mt}}$ mice, resulting in: (1) a reduction of tonic inward current (appearing as an outward current) and (2) an almost complete cessation of synaptic events (Fig. 9C). Amplitude and $20-80 \%$ rise time of the light response showed no significant differences between RIM $2 \alpha^{\mathrm{wt}}$ and RIM $2 \alpha^{\mathrm{mt}}$ mice (Fig. 9D). However, charge transfer values of the responses to light offset, which possibly represent evoked vesicle release from photoreceptors (Jackman et al., 2009), were significantly smaller in mutant mice. We have also used a train of full-field light stimuli switched on and off for 500 $\mathrm{ms}$ to investigate whether horizontal cell light responses undergo adaptation during repetitive stimulation. Light response amplitudes in both RIM $2 \alpha^{\mathrm{wt}}$ and RIM $2 \alpha^{\mathrm{mt}}$ mice showed no decline after 10 stimuli, but charge transfer values of off-light responses revealed a tendency to be smaller during the 10 stimuli, although they did not reach statistical significance in our measurements (Fig. 9E, F).

The findings from the ERG and, especially, the voltage-clamp recordings strongly argue for a mechanism of synaptic vesicle exocytosis from photoreceptor ribbon synapses that does not involve a RIM-Munc13-RAB3A complex, verifying an earlier study showing Munc13-independent vesicle priming at photoreceptor ribbon synapses (Cooper et al., 2012).

\section{Discussion}

RIM proteins are highly conserved active zone components of chemical synapses. Due to their interactions with various other proteins, they play important roles in the vesicle docking and/or priming process and in the synchronization of release during exocytosis by clustering and modulation of $\mathrm{Ca}^{2+}$ channels. In the present study, we examined the expression, localization, and function of RIMs at the specialized sensory photoreceptor ribbon synapse. As discussed below, our data provide important information for the current understanding of photoreceptor ribbon synaptic function.

\section{Absence of RIM1 from mouse photoreceptor ribbon synapses?}

In a preceding study, we reported the presence of both RIM1 and RIM2 at photoreceptor ribbon synapses, with RIM1 associated with the presynaptic ribbon and RIM2 with the arciform density of rod photoreceptors (tom Dieck et al., 2005). In the present study, we show that RIM1 is most likely absent from photoreceptor ribbon synapses and RIM2 is the major large RIM isoform present at

\footnotetext{
$\leftarrow$

(Figure legend continued.) $\boldsymbol{F}$, Peak light responses and off responses did not show significant adaptation during the course of stimulation as determined by fitting a linear regression line to the data points. Slope values for peak light responses were $-0.022 \pm 0.116$ and $-0.251 \pm$ 0.246 for RIM $1 \alpha^{\text {wt }}$ and RIM $1 \alpha^{\mathrm{mt}}$, respectively ( $p=0.4415$; unpaired $t$ test). Slope values for corresponding off responses were $0.120 \pm 0.099$ and $0.098 \pm 0.104$ ( $p=0.8764$; unpaired $t$ test). $n=11$ cells from 3 animals/genotype.
}

photoreceptor ribbon synapses. As we demonstrate, the most likely explanation for the discrepancy in the results of the two studies is that the antibodies against RIM1 used in the study by tom Dieck et al. (2005) cross-reacted with Piccolo/Piccolino.

The likely absence of RIM1 from photoreceptors at the protein and transcript level decisively influences our current thinking concerning vesicle tethering and priming at photoreceptor ribbon synapses. One hypothesized role of synaptic ribbons in sensory neurons is the recruitment and/or priming of synaptic vesicles via filamentous tethers to support high continuous release rates (Heidelberger, 2007; Frank et al., 2010; Matthews and Fuchs, 2010; Snellman et al., 2011; Hallermann and Silver, 2013). To date, the molecular identity of these tethers and the potential priming machinery at the synaptic ribbon are unknown. RIM1 has been discussed as the most likely candidate because it possesses the ability to interact with the vesicle-associated protein RAB3A and it has been reported to be located at the photoreceptor synaptic ribbon (Wang et al., 1997; Wang et al., 2001; tom Dieck et al., 2005; Limbach et al., 2011; Snellman et al., 2011; Tian et al., 2012, Hallermann and Silver, 2013). Moreover, cryoelectron tomographical experiments revealed reductions in vesicle tethering and vesicle numbers at RIMl $\alpha$-deficient active zones of cerebrocortical synapses (Fernández-Busnadiego et al., 2013).

Because we now show that RIM1 is probably not available as a vesicle tether at the photoreceptor synaptic ribbon, the question of which protein tethers synaptic vesicles to the photoreceptor synaptic ribbon is still open.

\section{Mouse photoreceptors predominantly express alternatively spliced RIM $2 \alpha$ and/or RIM2 $\beta$}

We found that RIM2, which localizes to the arciform density of the rod photoreceptor active zone (tom Dieck et al., 2005; RegusLeidig et al., 2010; this study), is the major large RIM isoform expressed in mouse photoreceptors. Using antibodies against different epitopes of RIM2 in combination with transcript analyses, we show that the majority of RIM2 in mouse photoreceptors lacks the N-terminal zinc finger domain and (part of) the RAB3A-binding domain. The analysis of a RIM $2 \alpha^{\mathrm{mt}}$ mouse line with a genetic deletion of RIM2 exon 5 supports this finding. This mutant mouse lacks RIM $2 \alpha$ in all tested brain regions (Schoch et al., 2006), but in photoreceptors, RIM2 protein levels were barely affected, indicating that the prevalent RIM2 transcript in photoreceptors lacks this exon.

\section{Interaction of photoreceptor RIM2 with ubMunc13-2 is not essential functionally in photoreceptor synaptic transmission} At conventional chemical synapses, RIM-Munc13-RAB3Ainteractions mediate docking/priming (Betz et al., 2001; Deng et al., 2011; Südhof, 2013). Characterization of the translation product of RIM $2 \alpha^{\Delta \mathrm{Ex2}-5}$, representing the entirety of RIM2 in wild-type mouse photoreceptors, revealed its inability to interact with the ribbon-synapse-specific ubMunc13-2 for vesicle priming and a weaker RAB3A binding than in the full-length RIM $2 \alpha$. To analyze the contribution of full-length RIM $2 \alpha$ to synaptic vesicle exocytosis at photoreceptor ribbon synapses, we characterized photoreceptor synaptic transmission in the absence of full-length RIM $2 \alpha$ in the RIM $2 \alpha^{\mathrm{mt}}$ retina. Because our voltageclamp recordings from postsynaptic horizontal cells of RIM $2 \alpha^{\text {wt }}$ and RIM $2 \alpha^{\mathrm{mt}}$ retinae did not reveal any significant functional differences between the cone photoreceptor ribbon synapses of the two genotypes, we conclude that the RIM-Munc13-RAB3A tripartite complex is not essential for synaptic vesicle exocytosis 
at photoreceptor ribbon synapses. The results from a previous study demonstrating that the absence of ubMunc13-2 does not impair vesicle priming and photoreceptor ribbon synaptic transmission significantly supports our hypothesis (Cooper et al., 2012).

In summary, our study shows that RIM2 is the prevalent large RIM isoform present at photoreceptor ribbon synapses. The majority of photoreceptor-RIM2 lacks the N-terminal zinc finger and (part of) the RAB3A-binding domain, which leads to its inability to interact with ubMunc13-2 and to decreased or absence of RAB3A binding. From our results and the results from a recent study on the function of RIMs at photoreceptor ribbon synapses (Grabner et al., 2015), we conclude that the role of RIM2 at photoreceptor ribbon synapses is different from RIM function in most other types of chemical synapses (Mittelstaedt et al., 2010; Jung et al., 2015). At the photoreceptor ribbon synaptic active zone, RIM2 is not essential for vesicle priming (this study) or for $\mathrm{Ca}^{2+}$ channel clustering, but it does act as a $\mathrm{Ca}^{2+}$ channel modulator (Grabner et al., 2015).

\section{References}

Alvarez-Baron E, Michel K, Mittelstaedt T, Opitz T, Schmitz F, Beck H, Dietrich D, Becker AJ, Schoch S (2013) RIM3 $\gamma$ and RIM4 $\gamma$ are key regulators of neuronal arborization. J Neurosci 33:824-839. CrossRef Medline

Andrews-Zwilling YS, Kawabe H, Reim K, Varoqueaux F, Brose N (2006) Binding to RAB3A-interacting molecule RIM regulates the presynaptic recruitment of Munc13-1 and ubMunc13-2. J Biol Chem 281:1972019731. CrossRef Medline

Babai N, Sendelbeck A, Regus-Leidig H, Fuchs M, Mertins J, Reim K, Brose N, Feigenspan A, Brandstätter JH (2016) Functional roles of complexin3 and complexin 4 at mouse photoreceptor ribbon synapses. J Neurosci 36:6651-6667. CrossRef Medline

Betz A, Thakur P, Junge HJ, Ashery U, Rhee JS, Scheuss V, Rosenmund C, Rettig J, Brose N (2001) Functional interaction of the active zone proteins Munc13-1 and RIM1 in synaptic vesicle priming. Neuron 30:183196. CrossRef Medline

Calakos N, Schoch S, Südhof TC, Malenka RC (2004) Multiple roles for the active zone protein RIM1alpha in late stages of neurotransmitter release. Neuron 42:889-896. CrossRef Medline

Cooper B, Hemmerlein M, Ammermüller J, Imig C, Reim K, Lipstein N, Kalla S, Kawabe H, Brose N, Brandstätter JH, Varoqueaux F (2012) Munc13independent vesicle priming at mouse photoreceptor ribbon synapses. J Neurosci 32:8040-8052. CrossRef Medline

Deng L, Kaeser PS, Xu W, Südhof TC (2011) RIM proteins activate vesicle priming by reversing autoinhibitory homodimerization of Munc13. Neuron 69:317-331. CrossRef Medline

Dick O, Hack I, Altrock WD, Garner CC, Gundelfinger ED, Brandstätter JH (2001) Localization of the presynaptic cytomatrix protein Piccolo at ribbon and conven-tional synapses in the rat retina: comparison with Bassoon. J Comp Neurol 439:224-234. CrossRef Medline

Feigenspan A, Babai N (2015) Functional properties of spontaneous excitatory currents and encoding of light/dark transitions in horizontal cells of the mouse retina. Eur J Neurosci 42:2615-2632. CrossRef Medline

Feigenspan A, Babai N (2017) Preparation of horizontal slices of adult mouse retina for electrophysiological studies. J Vis Exp 119.

Fernández-Busnadiego R, Asano S, Oprisoreanu AM, Sakata E, Doengi M, Kochovski Z, Zürner M, Stein V, Schoch S, Baumeister W, Lucić V (2013) Cryo-electron tomography reveals a critical role of RIM1 $\alpha$ in synaptic vesicle tethering. J Cell Biol 201:725-740. CrossRef Medline

Frank T, Rutherford MA, Strenzke N, Neef A, Pangršič T, Khimich D, Fejtova A, Fetjova A, Gundelfinger ED, Liberman MC, Harke B, Bryan KE, Lee A, Egner A, Riedel D, Moser T (2010) Bassoon and the synaptic ribbon organize $\mathrm{Ca} 2+$ channels and vesicles to add release sites and promote refilling. Neuron 68:724-738. CrossRef Medline

Frishman LJ (2006) Origins of the electroretinogram. In: Principles and practice of clinical electrophysiology of vision (Heckenlively JR, Arden GB, eds), pp 139-183. Cambridge, MA: MIT.

Fukuda M (2003) Distinct Rab binding specificity of Rim1, Rim2, rabphilin, and Noc2. Identification of a critical determinant of RAB3A/Rab27A recognition by Rim2. J Biol Chem 278:15373-15380. CrossRef Medline

Grabner CP, Gandini MA, Rehak R, Le Y, Zamponi GW, Schmitz F (2015) RIM1/2-mediated facilitation of Cav1.4 channel opening is required for Ca2+-stimulated release in mouse rod photoreceptors. J Neurosci 35: 13133-13147. CrossRef Medline

Hallermann S, Silver RA (2013) Sustaining rapid vesicular release at active zones: potential roles for vesicle tethering. Trends Neurosci 36:185-194. CrossRef Medline

Han Y, Kaeser PS, Südhof TC, Schneggenburger R (2011) RIM determines $\mathrm{Ca}^{2}+$ channel density and vesicle docking at the presynaptic active zone. Neuron 69:304-316. CrossRef Medline

Harazny J, Scholz M, Buder T, Lausen B, Kremers J (2009) Electrophysiological deficits in the retina of the DBA/2J mouse. Doc Ophthalmol 119: 181-197. CrossRef Medline

Heidelberger R (2007) Mechanisms of tonic, graded release: lessons from the vertebrate photoreceptor. J Physiol 585:663-667. CrossRef Medline

Jackman SL, Choi SY, Thoreson WB, Rabl K, Bartoletti TM, Kramer RH (2009) Role of the synaptic ribbon in transmitting the cone light response. Nat Neurosci 12:303-310. CrossRef Medline

Jung S, Oshima-Takago T, Chakrabarti R, Wong AB, Jing Z, Yamanbaeva G, Picher MM, Wojcik SM, Göttfert F, Predoehl F, Michel K, Hell SW, Schoch S, Strenzke N, Wichmann C, Moser T (2015) RAB3A-interacting molecules $2 \alpha$ and $2 \beta$ promote the abundance of voltage-gated $\mathrm{CaV} 1.3 \mathrm{Ca}^{2+}$ channels at hair cell active zones. Proc Natl Acad Sci U S A 112:E3141-E3149. CrossRef Medline

Kaeser PS, Kwon HB, Chiu CQ, Deng L, Castillo PE, Südhof TC (2008) RIM $1 \alpha$ and RIM $1 \beta$ are synthesized from distinct promoters of the RIM 1 gene to mediate differential but overlapping synaptic functions. J Neurosci 28:13435-13447. CrossRef Medline

Kaeser PS, Deng L, Wang Y, Dulubova I, Liu X, Rizo J, Südhof TC (2011) RIM proteins tether $\mathrm{Ca} 2+$ channels to presynaptic active zones via a direct PDZ-domain interaction. Cell 144:282-295. CrossRef Medline

Kaeser PS, Deng L, Fan M, Südhof TC (2012) RIM genes differentially contribute to organizing presynaptic release sites. Proc Natl Acad Sci U S A 109:11830-11835. CrossRef Medline

Limbach C, Laue MM, Wang X, Hu B, Thiede N, Hultqvist G, Kilimann MW (2011) Molecular in situ topology of Aczonin/Piccolo and associated proteins at the mammalian neurotransmitter release site. Proc Natl Acad Sci U S A 108:E392-E401. CrossRef Medline

Lonart G, Schoch S, Kaeser PS, Larkin CJ, Südhof TC, Linden DJ (2003) Phosphorylation of RIMlalpha by PKA triggers presynaptic long-term potentiation at cerebellar parallel fiber synapses. Cell 115:49-60. CrossRef Medline

Ma C, Li W, Xu Y, Rizo J (2011) Munc13 mediates the transition from the closed syntaxin-Munc18 complex to the SNARE complex. Nat Struct Mol Biol 18:542-549. CrossRef Medline

Matthews G, Fuchs P (2010) The diverse roles of ribbon synapses in sensory neurotransmission. Nat Rev Neurosci 11:812-822. CrossRef Medline

Mittelstaedt T, Alvaréz-Baron E, Schoch S (2010) RIM proteins and their role in synapse function. Biol Chem 391:599-606. CrossRef Medline

Regus-Leidig H, Tom Dieck S, Specht D, Meyer L, Brandstätter JH (2009) Early steps in the assembly of photoreceptor ribbon synapses in the mouse retina: the involvement of precursor spheres. J Comp Neurol 512:814824. CrossRef Medline

Regus-Leidig H, Specht D, Tom Dieck S, Brandstätter JH (2010) Stability of active zone components at the photoreceptor ribbon complex. Mol Vis 16:2690-2700. Medline

Regus-Leidig H, Ott C, Löhner M, Atorf J, Fuchs M, Sedmak T, Kremers J, Fejtová A, Gundelfinger ED, Brandstätter JH (2013) Identification and immunocytochemical characterization of Piccolino, a novel Piccolo splice variant selectively expressed at sensory ribbon synapses of the eye and ear. PLoS One 8:e70373. CrossRef Medline

Reim K, Wegmeyer H, Brandstätter JH, Xue M, Rosenmund C, Dresbach T, Hofmann K, Brose N (2005) Structurally and functionally unique complexins at retinal ribbon synapses. J Cell Biol 169:669-680. CrossRef Medline

Schoch S, Castillo PE, Jo T, Mukherjee K, Geppert M, Wang Y, Schmitz F, Malenka RC, Südhof TC (2002) RIMlalpha forms a protein scaffold for regulating neurotransmitter release at the active zone. Nature 415:321326. CrossRef Medline 
Schoch S, Mittelstaedt T, Kaeser PS, Padgett D, Feldmann N, Chevaleyre V, Castillo PE, Hammer RE, Han W, Schmitz F, Lin W, Südhof TC (2006) Redundant functions of RIM1alpha and RIM2alpha in $\mathrm{Ca}(2+)$-triggered neurotransmitter release. EMBO J 25:5852-5863. CrossRef Medline

Snellman J, Mehta B, Babai N, Bartoletti TM, Akmentin W, Francis A, Matthews G, Thoreson W, Zenisek D (2011) Acute destruction of the synaptic ribbon reveals a role for the ribbon in vesicle priming. Nat Neurosci 14:1135-1141. CrossRef Medline

Südhof TC (2013) Neurotransmitter release: the last millisecond in the life of a synaptic vesicle. Neuron 80:675-690. CrossRef Medline

Tian M, Xu CS, Montpetit R, Kramer RH (2012) RAB3A mediates vesicle delivery at photoreceptor ribbon synapses. J Neurosci 32:6931-6936. CrossRef Medline

Tom Dieck S, Altrock WD, Kessels MM, Qualmann B, Regus H, Brauner D, Fejtová A, Bracko O, Gundelfinger ED, Brandstätter JH (2005) Molecular dissection of the photoreceptor ribbon synapse: physical interaction of Bassoon and RIBEYE is essential for the assembly of the ribbon complex. J Cell Biol 168:825-836. CrossRef Medline

Wang X, Hu B, Zimmermann B, Kilimann MW (2001) Rim1 and rabphilin-3 bind RAB3A-GTP by composite determinants partially related through N-terminal alpha-helix motifs. J Biol Chem 276:32480-32488. CrossRef Medline

Wang Y, Südhof TC (2003) Genomic definition of RIM proteins: evolutionary amplification of a family of synaptic regulatory proteins. Genomics 81:126-137. CrossRef Medline

Wang Y, Okamoto M, Schmitz F, Hofmann K, Südhof TC (1997) Rim is a putative RAB3A effector in regulating synaptic-vesicle fusion. Nature 388: 593-598. CrossRef Medline

Yang X, Wang S, Sheng Y, Zhang M, Zou W, Wu L, Kang L, Rizo J, Zhang R, Xu T, Ma C (2015) Syntaxin opening by the MUN domain underlies the function of Munc13 in synaptic-vesicle priming. Nat Struct Mol Biol 22:547-554. CrossRef Medline 